\title{
Supersymmetric top and bottom squark production at hadron colliders
}

\author{
Wim Beenakker, ${ }^{a}$ Silja Brensing, ${ }^{b, c}$ Michael Krämer, ${ }^{d, b}$ Anna Kulesza, ${ }^{b}$ \\ Eric Laenen ${ }^{e, f, c}$ and Irene Niessen ${ }^{a}$ \\ ${ }^{a}$ Theoretical High Energy Physics, IMAPP, Radboud University Nijmegen, \\ P.O. Box 9010, NL-6500 GL Nijmegen, The Netherlands \\ ${ }^{b}$ Institut für Theoretische Teilchenphysik und Kosmologie, RWTH Aachen University, \\ D-52056 Aachen, Germany \\ ${ }^{c}$ Nikhef Theory Group, \\ Science Park 105, 1098 XG Amsterdam, The Netherlands \\ ${ }^{d}$ CERN, Physics Department, Theory Unit, \\ CH-1211 Geneva 23, Switzerland \\ e ITFA, University of Amsterdam, \\ Science Park 904, 1018 XE Amsterdam, The Netherlands \\ ${ }^{f}$ ITF, Utrecht University, \\ Leuvenlaan 4, 3584 CE Utrecht, The Netherlands \\ E-mail: W.Beenakker@science.ru.nl, brensing@physik.rwth-aachen.de, \\ mkraemer@physik.rwth-aachen.de, kulesza@physik.rwth-aachen.de, \\ Eric.Laenen@nikhef.nl, i.niessen@science.ru.nl
}

ABstRACT: The scalar partners of top and bottom quarks are expected to be the lightest squarks in supersymmetric theories, with potentially large cross sections at hadron colliders. We present predictions for the production of top and bottom squarks at the Tevatron and the LHC, including next-to-leading order corrections in supersymmetric QCD and the resummation of soft gluon emission at next-to-leading-logarithmic accuracy. We discuss the impact of the higher-order corrections on total cross sections and transverse-momentum distributions, and provide an estimate of the theoretical uncertainty due to scale variation and the parton distribution functions.

Keywords: QCD Phenomenology, Supersymmetry Phenomenology

ARXIV EPRINT: 1006.4771 


\section{Contents}

1 Introduction 1

2 Stop and sbottom pair production 3

2.1 Threshold resummation for the total inclusive cross section 5

2.2 Threshold resummation for the transverse-momentum distribution 6

3 Numerical results $\quad 9$

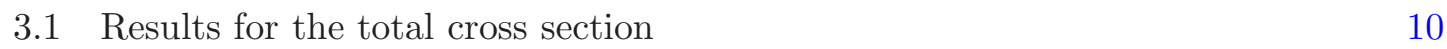

$\begin{array}{ll}3.2 & \text { Results for the transverse-momentum distribution } \\ \end{array}$

4 Conclusions $\quad 16$

$\begin{array}{ll}\text { A SUSY parameter dependence of stop and sbottom cross sections } & 19\end{array}$

\section{Introduction}

The search for supersymmetry $[1,2]$ is a central part of the physics program at the protonantiproton collider Tevatron with a centre-of-mass energy of $\sqrt{S}=1.96 \mathrm{TeV}$ and at the proton-proton collider LHC, which started operation in 2010 at $\sqrt{S}=7 \mathrm{TeV}$. In particular squarks and gluinos, the coloured supersymmetric particles, may be produced copiously in hadronic collisions. The hadroproduction of top squarks (stops) [3] is an important special case, since the strong Yukawa coupling between top quarks, stops and Higgs fields gives rise to potentially large mixing effects and mass splitting [4]. The same holds, albeit to a lesser extent, for bottom squarks (sbottoms). Moreover, if the scalar masses in unified supersymmetric theories are evolved from universal values at high scales down to low scales, the lighter of the stop mass eigenstates is generally driven to the lowest value in the entire squark mass spectrum. The search for the lightest stop therefore plays a special role in the quest to find signals of supersymmetry at hadron colliders.

Searches at LEP $[5,6]$ and the Tevatron [7]- [11] have placed lower limits on the lighter stop and sbottom mass eigenstates in the range between about $70-200 \mathrm{GeV}$, depending on the choice of supersymmetric parameters. The LHC will extend the range of sensitivity into the TeV-region $[12,13]$.

In the minimal supersymmetric extension of the Standard Model (MSSM) [14, 15] with R-parity conservation, stops are pair-produced at hadron colliders:

$$
p p / p \bar{p} \rightarrow \tilde{t}_{1} \overline{\tilde{t}}_{1}+X \text { and } \tilde{t}_{2} \overline{\tilde{t}}_{2}+X,
$$

where $\tilde{t}_{1}$ and $\tilde{t}_{2}$ denote the lighter and heavier mass eigenstate, respectively. The hadroproduction of mixed $\tilde{t}_{1} \overline{\tilde{t}}_{2}$ or $\tilde{t}_{2} \overline{\tilde{t}}_{1}$ final states is strongly suppressed since it can only proceed through electroweak channels or QCD-induced loop diagrams [3, 16, 17]. Sbottom 
hadroproduction is described in a completely analogous manner, so we will focus our discussion on stops. We will, however, comment on potential differences between stop and sbottom hadroproduction, and provide benchmark cross sections for sbottom production at the Tevatron and the LHC.

Accurate theoretical predictions for the stop-pair cross sections are crucial to derive exclusion limits [7]- [11] and, in the case of discovery, can be used to determine the stop masses and properties (see e.g. refs. [18-20]). The cross sections for the stop-pair production processes (1.1) have been calculated at next-to-leading order (NLO) in supersymmetric QCD (SUSY-QCD) [3]. The SUSY-QCD corrections significantly reduce the renormalization- and factorization-scale dependence and increase the cross section with respect to the leading-order ( $\mathrm{LO}$ ) predictions if the renormalization and factorization scales are chosen close to the stop mass. Electroweak corrections have been calculated as well $[21,22]$. Although they can be sizeable at large invariant masses and large transverse momenta, they are moderate for the inclusive stop cross section. The SUSY-QCD calculation of ref. [3] has been implemented in the public computer code Prospino [23, 24] and presently forms the theoretical basis for the stop mass limits obtained at the Tevatron.

A significant part of the large NLO SUSY-QCD corrections to squark hadroproduction can in general be attributed to the threshold region [3, 25] where the partonic centre-of-mass energy is close to the kinematic threshold for producing massive particles. In this region the NLO corrections are dominated by contributions from soft gluon emission off the coloured particles in the initial and final state and by Coulomb corrections due to the exchange of gluons between the massive particles in the final state. The soft-gluon corrections can be taken into account to all orders in perturbation theory by means of threshold resummation. A considerable amount of work has recently been devoted to the calculation of threshold logarithms for total gluino and squark cross sections [26]- [31]. Final-state stops are excluded in these calculations and all other squark flavours, the so-called light-flavour squarks, are treated as being mass degenerate, neglecting possible mixing effects.

In this work, we extend the previous analyses of threshold resummation for the hadroproduction of gluinos and mass-degenerate light-flavour squarks at next-to-leading logarithmic (NLL) accuracy [26, 28, 31]. Firstly, we consider the hadroproduction of stops and non-mass-degenerate sbottoms. Secondly, we study the impact of NLO and NLL corrections on the transverse-momentum distributions. Since theoretical predictions for differential distributions are input to the experimental analyses, it is important to assess how the shape of the distributions is affected by higher-order corrections. The threshold resummation for transverse-momentum distributions has been studied extensively for Standard-Model processes, see e.g. refs. [32] - [38], but not yet for SUSY processes.

The paper is structured as follows. In section 2 we review the basic features of stop and sbottom hadroproduction, and we briefly discuss the application of threshold resummation to the transverse-momentum distribution. section 2 also contains the specific stop-pair formulae that enter the calculation of the resummed cross sections. State-of-the-art SUSYQCD predictions for stop hadroproduction at the Tevatron and the LHC, including NLO corrections and NLL threshold resummation, are presented in section 3. We discuss the impact of the NLO+NLL corrections on total cross sections and transverse-momentum 

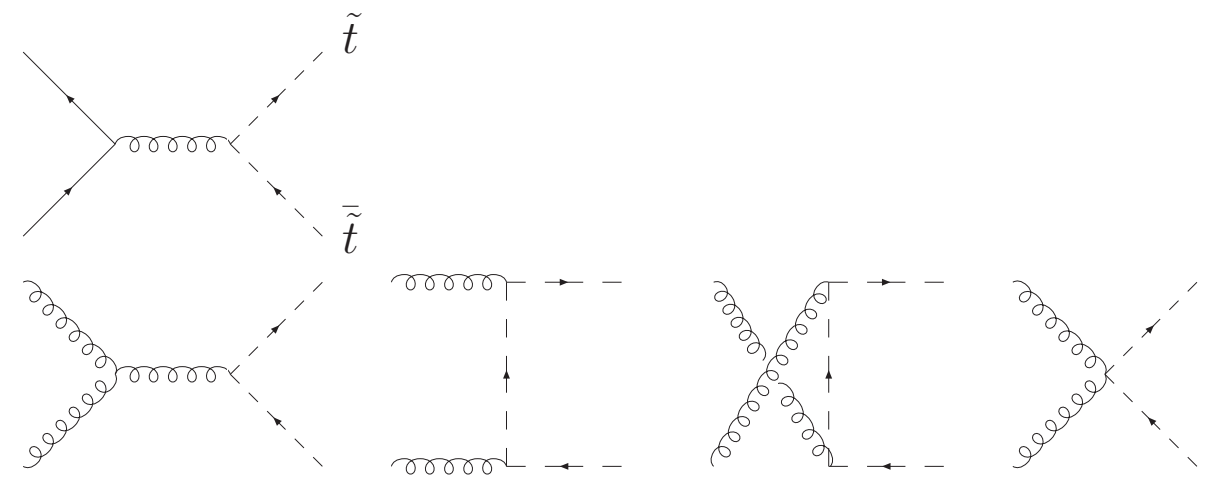

Figure 1. Leading-order Feynman diagrams for the production of stop pairs through quarkantiquark annihilation (first line) and gluon-gluon fusion (second line).

distributions and provide an estimate of the theoretical uncertainty due to scale variation and the parton distribution functions. We conclude in section 4. The dependence of the stop and sbottom cross sections on the choice of supersymmetric parameters can be found in the appendix, where also some predictions for specific benchmark scenarios are given.

\section{Stop and sbottom pair production}

Let us first review some basic features of the stop and sbottom pair-production cross sections. At LO the hadroproduction of stop pairs proceeds through quark-antiquark annihilation and gluon-gluon fusion:

$$
\begin{aligned}
& q \bar{q} \rightarrow \tilde{t}_{1} \overline{\tilde{t}}_{1} \quad \text { and } \quad \tilde{t}_{2} \overline{\tilde{t}}_{2}, \\
& g g \rightarrow \tilde{t}_{1} \tilde{\tilde{t}}_{1} \quad \text { and } \quad \tilde{t}_{2} \overline{\tilde{t}}_{2} .
\end{aligned}
$$

The corresponding Feynman diagrams are shown in figure 1. In contrast to the hadroproduction of light-flavour squarks, no $t$-channel gluino-exchange graph occurs in the quarkantiquark channel. In such a $t$-channel graph the initial-state quarks should have the same flavour as the final-state squarks, but since top quarks are excluded as initial-state partons, the gluino-exchange graph is absent.

The mass eigenstates $\tilde{t}_{1}$ and $\tilde{t}_{2}$ are related to the weak interaction eigenstates $\tilde{t}_{L}$ and $\tilde{t}_{R}$ through mixing: $\tilde{t}_{1}=\tilde{t}_{L} \cos \theta_{\tilde{t}}+\tilde{t}_{R} \sin \theta_{\tilde{t}}$ and $\tilde{t}_{2}=-\tilde{t}_{L} \sin \theta_{\tilde{t}}+\tilde{t}_{R} \cos \theta_{\tilde{t}}$. The masses $m_{\tilde{t}_{1}}, m_{\tilde{t}_{2}}$ and the mixing angle $\theta_{\tilde{t}}$ are obtained from diagonalizing the stop mass matrix and are determined by Standard-Model and soft-supersymmetry-breaking parameters [4]. As mentioned in the introduction, mixed pairs $\tilde{t}_{1} \overline{\tilde{t}}_{2}$ or $\tilde{t}_{2} \overline{\tilde{t}}_{1}$ cannot be produced in lowest

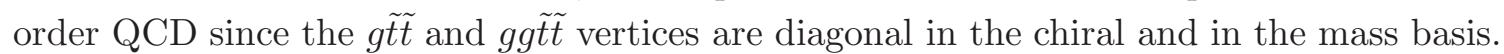

The description of sbottom hadroproduction $p p / p \bar{p} \rightarrow \tilde{b}_{1} \overline{\tilde{b}}_{1}$ and $\tilde{b}_{2} \overline{\tilde{b}}_{2}$ is completely analogous to that of stop production. The only differences occur in the $b \bar{b} \rightarrow \tilde{b} \tilde{b}$ channel, where the initial-state bottom quarks do allow a $t$-channel gluino-exchange graph that gives rise to extra contributions. As will be discussed below, these contributions lead to a less-suppressed LO threshold behaviour than the $s$-channel gluon-exchange contributions. 
However, we shall demonstrate in the appendix that their numerical impact on the hadronic cross sections is negligible. Thus, for all practical purposes, the LO and higher-order crosssection predictions obtained for stop-pair production apply also to sbottom-pair production if the input parameters, i.e. masses and mixing angles, are modified accordingly.

When decomposed into $s$-channel colour states, the LO partonic cross sections for the subprocesses (2.1) read in generic notation:

$$
\begin{aligned}
\sigma_{q \bar{q} \rightarrow \tilde{\tilde{t}}, \mathbf{1}}^{(0)} & 0 \\
\sigma_{q \bar{q} \rightarrow \tilde{\tilde{t}}, \mathbf{8}}^{(0)}= & \frac{\alpha_{\mathrm{s}}^{2} \pi\left(N_{c}^{2}-1\right)}{12 N_{c}^{2} s} \beta^{3} \\
\sigma_{g g \rightarrow \tilde{\tilde{t}}, \mathbf{1}}^{(0)}= & \frac{\alpha_{\mathrm{s}}^{2} \pi}{N_{c}\left(N_{c}^{2}-1\right) s}\left[\left(\frac{1}{2}+\frac{2 m^{2}}{s}\right) \beta+\left(\frac{2 m^{2}}{s}-\frac{4 m^{4}}{s^{2}}\right) \log \left(\frac{1-\beta}{1+\beta}\right)\right], \\
\sigma_{g g \rightarrow \tilde{\tilde{t}}, \mathbf{8}}^{(0)}= & \frac{N_{c}^{2}-4}{2} \sigma_{g g \rightarrow \tilde{t} \tilde{t}, \mathbf{1}}^{(0)} \\
& +\frac{\alpha_{\mathrm{s}}^{2} \pi N_{c}}{\left(N_{c}^{2}-1\right) s}\left[\left(\frac{1}{12}+\frac{8 m^{2}}{3 s}\right) \beta+\left(\frac{m^{2}}{s}+\frac{2 m^{4}}{s^{2}}\right) \log \left(\frac{1-\beta}{1+\beta}\right)\right]
\end{aligned}
$$

where $\alpha_{\mathrm{s}}$ is the strong coupling, $s$ the invariant partonic energy and $N_{c}$ the number of colours. The colour labels 1 and $\mathbf{8}$ refer to the familiar singlet and octet colour states in $\mathrm{SU}(3)$, but all analytic results in this paper are derived for a general $\mathrm{SU}\left(N_{c}\right)$-theory in order to make the colour structure more transparent. Also note that we use a generic notation for the final-state particles and the associated kinematics: $\tilde{t}$ can be either of the two stop mass eigenstates, with $m$ being the corresponding mass and $\beta=\sqrt{1-4 m^{2} / \mathrm{s}}$ the corresponding velocity.

The expressions (2.4) and (2.5) for the gluon-gluon fusion cross section agree with the corresponding expression for light-flavour squark production, cf. ref. [25]. However, the quark-antiquark annihilation contribution (2.3) is different due to the absence of the $t$-channel gluino-exchange graph. As a consequence, the LO cross section for $q \bar{q} \rightarrow \tilde{t} \bar{t}$ proceeds through $s$-channel gluon exchange only and is proportional to $\beta^{3}$, as opposed to $\beta$ for other squark flavours. This $\beta^{3}$ behaviour is the combined effect of the standard phasespace suppression factor $\beta$ and an additional $P$-wave suppression $\propto \beta^{2}$ near threshold: the pair of scalar particles needs to be produced in a $P$-wave state to balance the spin of the intermediate gluon.

Note that the LO cross sections (2.2)-(2.5) only depend on the mass $m$ of the produced stops and not on any other supersymmetric parameters. At NLO, however, the stop mixing angle $\theta_{\tilde{t}}$ enters through corrections involving the $t \tilde{t} \tilde{g}$ vertex and the four-squark couplings. As a result, already the analytical expressions for the $\tilde{t}_{1} \overline{\tilde{t}}_{1}$ and $\tilde{t}_{2} \overline{\tilde{t}}_{2}$ NLO cross sections are different. Furthermore, virtual corrections involving squark and gluino loops introduce a dependence of the stop-pair cross section on the masses of the squarks and the gluino. The dependence on all these other supersymmetric parameters turns out to be mild, as illustrated in the appendix.

In the threshold region the NLO stop-pair cross section is dominated by soft gluon emission, which leads to corrections $\propto \log ^{i} \beta(i=1,2)$, and Coulomb corrections $\propto 1 / \beta$. 
In the notation of ref. [3] the threshold behaviour of the total NLO cross sections reads: ${ }^{1}$

$$
\begin{aligned}
\underset{q \bar{q} \rightarrow \tilde{t} \tilde{\bar{t}}}{(1, \mathrm{thr})}= & \frac{\pi \alpha_{\mathrm{S}}^{2}\left(\mu^{2}\right)}{48 m^{2}} \frac{N_{c}^{2}-1}{N_{c}^{2}} \beta^{3}\left(1+4 \pi \alpha_{\mathrm{s}}\left(\mu^{2}\right)\left\{\frac{2 C_{F}-C_{A}}{16 \beta}-\frac{C_{A}}{4 \pi^{2}} \log \left(8 \beta^{2}\right)\right.\right. \\
& \left.\left.+\frac{2 C_{F}}{4 \pi^{2}}\left[\log ^{2}\left(8 \beta^{2}\right)-\frac{16}{3} \log \left(8 \beta^{2}\right)-\log \left(8 \beta^{2}\right) \log \left(\frac{\mu^{2}}{m^{2}}\right)\right]\right\}\right)
\end{aligned}
$$

and

$$
\begin{aligned}
\underset{g g \rightarrow \tilde{t} \tilde{t}}{(1, \mathrm{thr})}= & \frac{\pi \alpha_{\mathrm{S}}^{2}\left(\mu^{2}\right)}{16 m^{2}} \frac{N_{c}^{2}-2}{N_{c}\left(N_{c}^{2}-1\right)} \beta\left(1+4 \pi \alpha_{\mathrm{S}}\left(\mu^{2}\right)\left\{\frac{2 C_{F}-\frac{N_{c}^{2}-4}{N_{c}^{2}-2} C_{A}}{16 \beta}-\frac{N_{c}^{2}-4}{N_{c}^{2}-2} \frac{C_{A}}{4 \pi^{2}} \log \left(8 \beta^{2}\right)\right.\right. \\
& \left.\left.+\frac{2 C_{A}}{4 \pi^{2}}\left[\log ^{2}\left(8 \beta^{2}\right)-4 \log \left(8 \beta^{2}\right)-\log \left(8 \beta^{2}\right) \log \left(\frac{\mu^{2}}{m^{2}}\right)\right]\right\}\right) .
\end{aligned}
$$

Here $\mu$ denotes the factorization and renormalization scales, which we keep equal in this analysis. The first line in both expressions contains the gluon corrections that can be attributed to the final-state particles, while the terms in the second line, proportional to either $C_{F}=\left(N_{c}^{2}-1\right) /\left(2 N_{c}\right)$ or $C_{A}=N_{c}$ correspond to initial-state radiation from a quark or a gluon line, respectively. The final-state radiation consists of two parts. The logarithmic soft-emission terms are proportional to the colour charge of the final state [26, 29, 31, 39] and are therefore absent for the singlet colour state and proportional to $C_{A}$ for the octet colour state. As a result, in the expression for the gluon-gluon fusion channel these terms are multiplied by $\left(N_{c}^{2}-4\right) /\left(N_{c}^{2}-2\right)$, which is the ratio of the colour-octet and the total cross section at threshold (cf. [39]). The Coulomb corrections receive contributions with colour factors $C_{F}$ and the total colour charge of the final state. Note, finally, that the threshold behaviour of the cross section is determined by QCD dynamics and does not involve any supersymmetric parameters other than the mass of the produced stops.

\subsection{Threshold resummation for the total inclusive cross section}

In this paper we shall improve the NLO prediction for the hadroproduction of stop and sbottom pairs [3] by resumming the leading and next-to-leading threshold logarithms of the form

$$
\alpha_{\mathrm{s}}^{n} \log ^{m} \beta^{2}, \quad m \leq 2 n
$$

to all orders, and to next-to-leading logarithmic (NLL) accuracy. The resummation formalism developed for heavy-quark production [39-41] can be applied directly to squarkantisquark production as the colour structure is identical for both cases. The resummation is performed in the space of Mellin moments ( $N$-space) following the procedure outlined in refs. [26, 28, 31], where the hadroproduction of light-flavour squarks and gluinos at NLL accuracy has been considered.

From the viewpoint of threshold resummation there is a notable difference between the case of stops and light-flavour squarks, arising from the extra $\beta^{2}$ suppression of the

\footnotetext{
${ }^{1}$ Ref. [3] contains a misprint in the $\log \left(8 \beta^{2}\right)$-coefficient for the $q \bar{q}$-channel. In eq. (9) of [3] the coefficient $-107 /\left(36 \pi^{2}\right)$ should be replaced by $-155 /\left(36 \pi^{2}\right)$.
} 
quark-antiquark annihilation cross section $\sigma_{q \bar{q} \rightarrow \tilde{\bar{t}}}$ near threshold. In $N$-space this effectively produces an extra factor $1 / N$ compared to the light-flavour squark case, resulting in leading contributions $\propto \log ^{i}(N) / N$ instead of $\log ^{i}(N)$. For the seemingly analogous case of threshold resummation for the deep-inelastic structure function $F_{L}$, which has a leading behaviour of the type $\log ^{i}(N) / N$ as well, differences from the expected NLL resummation structure were revealed in refs. [42] and [43]. However, the NLL resummation procedure developed for light-flavour squarks applies to stop production as well. In the case of $F_{L}$ the extra factor $1 / N$ with respect to the $N$ dependence of the structure function $F_{2}$ arises due to a special initial-state jet function $[44,45]$ associated with the longitudinal projection. In contrast, in our case the $\log ^{i}(N) / N$ dependence arises from projecting onto the $P$-wave final state, which does not depend on the initial state jet function. Moreover, at $\mathcal{O}\left(\alpha_{\mathrm{s}}\right)$ leading and subleading $\log (N) / N$ corrections can be computed from the one-loop calculations, and they do exhibit the pattern expected from NLL threshold resummation. Finally, in view of the different threshold behaviour of the $q \bar{q}$ and $g g$ channels one might worry about the possibility that these channels mix in the NLL threshold resummation as a result of soft-quark emissions, which is not the case for top-quark and light-flavour squark production. However, we have checked that to NLL accuracy no such mixing occurs at $\mathcal{O}\left(\beta^{3}\right)$. Based on these observations we are confident that the expressions of refs. [26, 28, 31] can be applied to inclusive stop-pair production as well.

The new elements that enter the NLL resummed cross section for stop pair production are the LO partonic cross sections in $N$-space (indicated by a tilde), decomposed into $s$-channel singlet and octet colour states. They are given by:

$$
\begin{aligned}
& \tilde{\sigma}_{q \bar{q} \rightarrow \tilde{t} \tilde{t}, \mathbf{1}}^{(0)}(N)=0 \\
& \tilde{\sigma}_{q \bar{q} \rightarrow \tilde{t} \bar{t}, \mathbf{8}}^{(0)}(N)=\frac{\alpha_{\mathrm{s}}^{2} \pi^{3 / 2}}{64 m^{2}} \frac{N_{c}^{2}-1}{N_{c}^{2}} \frac{\Gamma(N+1)}{\Gamma(N+7 / 2)}, \\
& \tilde{\sigma}_{g g \rightarrow \tilde{t} \bar{t}, \mathbf{1}}^{(0)}(N)=\frac{\alpha_{\mathrm{s}}^{2} \pi^{3 / 2}}{16 m^{2}} \frac{1}{N_{c}\left(N_{c}^{2}-1\right)} \frac{N(N+3)+4}{(N+2)(N+3)} \frac{\Gamma(N+1)}{\Gamma(N+5 / 2)}, \\
& \tilde{\sigma}_{g g \rightarrow \tilde{t} \tilde{t}, \mathbf{8}}^{(0)}(N)=\frac{N_{c}^{2}-4}{2} \sigma_{g g \rightarrow \tilde{t} \tilde{t}, \mathbf{1}}^{(0)}(N)+\frac{\alpha_{\mathrm{s}}^{2} \pi^{3 / 2}}{64 m^{2}} \frac{N_{c}}{N_{c}^{2}-1} \frac{N(N+3)+4}{(N+2)(N+3)} \frac{\Gamma(N+1)}{\Gamma(N+7 / 2)} .
\end{aligned}
$$

The results for the gluon-gluon channels (2.11) and (2.12) agree with those presented in [28] for light-flavour squarks and $N_{c}=3$, and are reproduced here for completeness for general $\mathrm{SU}\left(N_{c}\right)$. Note that the results in [28] include an additional factor $2 n_{f}$ from the summation over the $L$ and $R$ squarks of $n_{f}$ light flavours.

\subsection{Threshold resummation for the transverse-momentum distribution}

Similar to the inclusive cross section, soft-gluon corrections can also dominate the transverse-momentum distribution of the stops if the production takes place sufficiently close to the edge of phase space. We now briefly review the general construction of threshold-resummed transverse-momentum distributions (cf. [32]-[38]) and its application to stop-pair production. 
We start with the general framework applicable to the hadroproduction of a pair of massive coloured particles. Assuming that one of the final-state particles carries a transverse momentum $p_{T}$, the minimal energy necessary to produce the system is $2 m_{T}$, where the transverse mass $m_{T}$ is defined by

$$
m_{T}=\sqrt{m^{2}+p_{T}^{2}} .
$$

For the $p_{T}$-distribution the dominant contributions originating from soft gluon emission have again the structure (2.8), with the variable $\beta$ replaced by (cf. [46])

$$
\beta_{T}=\sqrt{1-4 m_{T}^{2} / s} .
$$

Like in the case of the total inclusive cross section, the resummation of the logarithmic threshold corrections to $p_{T}$-distributions takes place in the space of Mellin moments. However, in this case the Mellin transform is taken with respect to the scaling variable $\hat{x}_{T}^{2}=4 m_{T}^{2} / s$, i.e.

$$
\frac{d \tilde{\sigma}_{i j \rightarrow k l}}{d p_{T}}\left(N, p_{T}, \mu^{2}\right) \equiv \int_{0}^{1} d \hat{x}_{T}^{2}\left(\hat{x}_{T}^{2}\right)^{N-1} \frac{d \sigma_{i j \rightarrow k l}}{d p_{T}}\left(\hat{x}_{T}^{2}, p_{T}, \mu^{2}\right)
$$

for a generic partonic subprocess $i j \rightarrow k l$.

In $N$-moment space the resummed partonic $p_{T}$-distribution for the hadroproduction of two massive coloured particles is given by

$$
\begin{aligned}
\frac{d \tilde{\sigma}_{i j \rightarrow k l}^{(\mathrm{res})}}{d p_{T}}\left(N, p_{T}, \mu^{2}\right)= & \sum_{I} \frac{d \tilde{\sigma}_{i j \rightarrow k l, I}^{(0)}}{d p_{T}}\left(N, p_{T}, \mu^{2}\right) C_{i j \rightarrow k l, I}\left(N, p_{T}, \mu^{2}\right) \\
& \times \Delta_{i}\left(N+1, Q^{2}, \mu^{2}\right) \Delta_{j}\left(N+1, Q^{2}, \mu^{2}\right) \Delta_{i j \rightarrow k l, I}^{(\mathrm{s})}\left(N+1, Q^{2}, \mu^{2}\right),
\end{aligned}
$$

with $Q=2 m_{T}$ representing the resummation scale and the index $I$ indicating all possible colour states of the hard scattering. To NLL accuracy, the coefficients $C_{i j \rightarrow k l, I}\left(N, p_{T}, \mu^{2}\right)$ are equal to 1 for all channels and colour structures. The functions $\Delta_{i}$ and $\Delta_{j}$ sum the effects of the (soft-)collinear radiation from the incoming partons. They are process- and colour-independent, and are therefore a universal ingredient in all threshold-resummed cross sections. Explicit expressions for the $\Delta_{i}$ can for instance be found in ref. [28]. The function $\Delta_{i j \rightarrow k l, I}^{(\mathrm{s})}$ accounts for soft wide-angle emission and depends on the production process and the colour channel. At NLL accuracy it is given by

$$
\Delta_{i j \rightarrow k l, I}^{(\mathrm{s})}\left(N, Q^{2}, \mu^{2}\right)=\exp \left[\int_{\mu}^{Q / N} \frac{d q}{q} \frac{\alpha_{\mathrm{s}}(q)}{\pi} D_{i j \rightarrow k l, I}\right] .
$$

The values of the coefficients $D_{i j \rightarrow k l, I}$ follow from the threshold limit of the one-loop soft anomalous-dimension matrix in the way described e.g. in ref. [31]. If an $s$-channel colour basis is used, the soft anomalous-dimension matrix for the $p_{T}$-distribution becomes diagonal in colour space in the threshold limit $\beta_{T} \rightarrow 0$, leading to the colour-diagonal form of the resummation formula given in eq. (2.16). This is similar to threshold resummation 
for the total cross section, where the soft anomalous-dimension matrix becomes diagonal in colour space in the corresponding threshold limit $\beta \rightarrow 0$. Since the threshold limit is defined differently for the total inclusive cross section and the $p_{T}$-distribution, the values of the $D$ coefficients are different as well; in particular, the $D$-coefficients now carry $p_{T}$-dependence.

For the stop-pair production processes we have

$$
\begin{aligned}
& D_{q \bar{q} / g g \rightarrow \tilde{t} \tilde{t}, \mathbf{1}}=-2 C_{F}\left(\operatorname{Re} L_{\bar{\beta}_{T}}+1\right), \\
& D_{q \bar{q} / g g \rightarrow \tilde{t} \tilde{t}, \mathbf{8}}=-2 C_{F}\left(\operatorname{Re} L_{\bar{\beta}_{T}}+1\right)+C_{A}\left[\log \left(\frac{m_{T}^{2}}{m^{2}}\right)+\operatorname{Re} L_{\bar{\beta}_{T}}\right],
\end{aligned}
$$

with

$$
\operatorname{Re} L_{\bar{\beta}_{T}}=\frac{1+\bar{\beta}_{T}^{2}}{2 \bar{\beta}_{T}} \log \left(\frac{1-\bar{\beta}_{T}}{1+\bar{\beta}_{T}}\right)
$$

and

$$
\bar{\beta}_{T}=\lim _{\hat{x}_{T}^{2} \rightarrow 1} \beta_{T}=\sqrt{1-m^{2} / m_{T}^{2}} .
$$

These expressions agree with those obtained for heavy-quark production in the context of joint resummation [38].

In addition to the soft radiative factor, the other new elements which have to be calculated in order to obtain resummed predictions from eq. (2.16) are the colour-decomposed LO $p_{T}$-distributions in $N$-moment space. They are obtained from

$$
\begin{aligned}
& \frac{d \sigma_{q \bar{q} \rightarrow \tilde{t} \tilde{t}, \mathbf{1}}^{(0)}}{d p_{T}}=0, \\
& \frac{d \sigma_{q \bar{q} \rightarrow \tilde{t} \tilde{t}, \mathbf{8}}^{(0)}}{d p_{T}}=\frac{\alpha_{\mathrm{s}}^{2} \pi}{s^{3}} \frac{2\left(N_{c}^{2}-1\right)}{N_{c}^{2}} \frac{p_{T}^{3}}{\beta_{T}}, \\
& \frac{d \sigma_{g g \rightarrow \tilde{t} \tilde{t}, \mathbf{1}}^{(0)}}{d p_{T}}=\frac{\alpha_{\mathrm{s}}^{2} \pi}{s^{2}} \frac{2}{N_{c}\left(N_{c}^{2}-1\right)} \frac{p_{T}}{\beta_{T}} \frac{m^{4}+p_{T}^{4}}{m_{T}^{4}}, \\
& \frac{d \sigma_{g g \rightarrow \tilde{t} \tilde{t}, \mathbf{8}}^{(0)}}{d p_{T}}=\frac{N_{c}^{2}-4}{2} \frac{d \sigma_{g g \rightarrow \tilde{t}, \mathbf{1}}^{(0)}}{d p_{T}}+\frac{\alpha_{\mathrm{s}}^{2} \pi}{s^{2}} \frac{N_{c}}{N_{c}^{2}-1} p_{T} \beta_{T} \frac{m^{4}+p_{T}^{4}}{m_{T}^{4}} .
\end{aligned}
$$

Taking a Mellin transform of $(2.22)-(2.25)$ with respect to the threshold variable $\hat{x}_{T}^{2}$ one finds

$$
\begin{aligned}
& \frac{d \tilde{\sigma}_{q \bar{q} \rightarrow \tilde{t} \tilde{t}, \mathbf{1}}^{(0)}}{d p_{T}}(N)=0, \\
& \frac{d \tilde{\sigma}_{q \bar{q} \rightarrow \tilde{t} \bar{t}, 8}^{(0)}}{d p_{T}}(N)=\frac{\alpha_{\mathrm{s}}^{2} \pi^{3 / 2}}{32} \frac{N_{c}^{2}-1}{N_{c}^{2}} \frac{\Gamma(N+3)}{\Gamma(N+7 / 2)} \frac{p_{T}^{3}}{m_{T}^{6}}, \\
& \frac{d \tilde{\sigma}_{g g \rightarrow \tilde{t} \tilde{t}, 1}^{(0)}}{d p_{T}}(N)=\frac{\alpha_{\mathrm{s}}^{2} \pi^{3 / 2}}{8} \frac{1}{N_{c}\left(N_{c}^{2}-1\right)} \frac{\Gamma(N+2)}{\Gamma(N+5 / 2)} \frac{p_{T}\left(m^{4}+p_{T}^{4}\right)}{m_{T}^{8}}, \\
& \frac{d \tilde{\sigma}_{g g \rightarrow \tilde{t} \tilde{t}, \mathbf{8}}^{(0)}}{d p_{T}}(N)=\frac{N_{c}^{2}-4}{2} \frac{d \tilde{\sigma}_{g g \rightarrow \tilde{t} \tilde{t}, \mathbf{1}}^{(0)}}{d p_{T}}(N)+\frac{\alpha_{\mathrm{s}}^{2} \pi^{3 / 2}}{32} \frac{N_{c}}{N_{c}^{2}-1} \frac{\Gamma(N+2)}{\Gamma(N+7 / 2)} \frac{p_{T}\left(m^{4}+p_{T}^{4}\right)}{m_{T}^{8}} .
\end{aligned}
$$


Having calculated the resummed partonic expression in $N$-space, eq. (2.16), the resummed hadronic $p_{T}$-distribution is obtained by the inverse Mellin transform

$$
\begin{aligned}
\frac{d \sigma_{h_{1} h_{2} \rightarrow k l}^{(\mathrm{res}}}{d p_{T}}\left(x_{T}^{2}, p_{T}, \mu^{2}\right)= & \sum_{i, j=q, \bar{q}, g} \int_{\mathrm{CT}} \frac{d N}{2 \pi i}\left(x_{T}^{2}\right)^{-N} \tilde{f}_{i / h_{1}}\left(N+1, \mu^{2}\right) \tilde{f}_{j / h_{2}}\left(N+1, \mu^{2}\right) \\
& \times \frac{d \tilde{\sigma}_{i j \rightarrow k l}^{(\mathrm{res})}}{d p_{T}}\left(N, p_{T}, \mu^{2}\right)
\end{aligned}
$$

where $x_{T}^{2}=4 m_{T}^{2} / S$ is the hadronic scaling variable. The functions $\tilde{f}_{i / h_{1}}$ and $\tilde{f}_{j / h_{2}}$ are the Mellin moments of the parton distribution functions for the initial-state hadrons $h_{1}$ and $h_{2}$. In order to retain the information contained in the NLO $p_{T}$-distributions [3], the NLO and NLL results are combined through a matching procedure that avoids double counting of the logarithmic terms in the following way:

$$
\begin{aligned}
\frac{d \sigma_{h_{1} h_{2} \rightarrow k l}^{(\mathrm{NLO}+\mathrm{NLL})}}{d p_{T}}\left(x_{T}^{2}, p_{T}, \mu^{2}\right)= & \frac{d \sigma_{h_{1} h_{2} \rightarrow k l}(\mathrm{NLO})}{d p_{T}}\left(x_{T}^{2}, p_{T}, \mu^{2}\right) \\
& +\sum_{i, j=q, \bar{q}, g} \int_{\mathrm{CT}} \frac{d N}{2 \pi i}\left(x_{T}^{2}\right)^{-N} \tilde{f}_{i / h_{1}}\left(N+1, \mu^{2}\right) \tilde{f}_{j / h_{2}}\left(N+1, \mu^{2}\right) \\
& \times\left[\frac{d \tilde{\sigma}_{i j \rightarrow k l}^{(\mathrm{res})}}{d p_{T}}\left(N, p_{T}, \mu^{2}\right)-\left.\frac{d \tilde{\sigma}_{i j \rightarrow k l}^{(\mathrm{res})}}{d p_{T}}\left(N, p_{T}, \mu^{2}\right)\right|_{(\mathrm{NLO})}\right],(2.31
\end{aligned}
$$

where $\left.\left(d \tilde{\sigma}^{\text {(res) }} / d p_{T}\right)\right|_{(\mathrm{NLO})}$ represents the perturbative expansion of the NLL $p_{T^{-}}$ distribution (2.16) truncated at the order of $\alpha_{\mathrm{s}}$ associated with NLO. To evaluate the inverse Mellin transform in eqs. (2.30) and (2.31) we adopt the "minimal prescription" of ref. [47] for the integration contour CT.

\section{$3 \quad$ Numerical results}

We present numerical results for the NLL-resummed cross sections and transverse-momentum distributions matched with the complete NLO results for stop-pair production at the Tevatron $(\sqrt{S}=1.96 \mathrm{TeV})$ and the $\mathrm{LHC}(\sqrt{S}=7$ and $14 \mathrm{TeV})$. The matching procedures for the total cross section and for the transverse-momentum distribution are described in ref. [31] and in eq. (2.31), respectively. We use the notation NLO+NLL for matched quantities in the following. The NLO corrections are calculated using Prospino [23, 24], based on the calculation presented in ref. [3]. The QCD coupling $\alpha_{\mathrm{s}}$ and the parton distribution functions (pdfs) at NLO are defined in the $\overline{\mathrm{MS}}$ scheme with five active flavours. The mass of the stop is renormalized in the on-shell scheme and the SUSY particles are decoupled from the running of $\alpha_{\mathrm{s}}$ and the pdfs. Since mixing enters explicitly only through higher-order diagrams, the angle $\theta_{\tilde{t}}$ need not be renormalized and one can use the lowestorder expression derived from the stop mass matrix.

As our default, hadronic total cross sections and transverse-momentum distributions are obtained with the 2008 NLO MSTW pdfs [48] and the corresponding $\alpha_{\mathrm{s}}\left(M_{Z}\right)=0.120$. 
The NLL corrections are convoluted with pdfs in Mellin space, derived with the program PEGASUS [50] based on the MSTW parametrization at the initial factorization scale. For the total hadronic cross sections, we have also used the method of ref. [51] to evaluate the NLL cross section with standard parametrizations of pdfs in $x$-space. We find however a much better numerical stability when the total NLL cross sections are evaluated with Mellin-space pdfs.

Beyond LO the cross section does not only depend on the stop mass, but also on the gluino mass $m_{\tilde{g}}$, the average mass of the first and second generation squarks $m_{\tilde{q}}$ and the mixing angle $\theta_{\tilde{t}}$. For this reason we have adopted the SPS1a' benchmark scenario [52] for our numerical analysis. Taking a top-quark mass of $m_{t}=172.5 \mathrm{GeV}[53]$ and $\alpha_{\mathrm{s}}\left(M_{Z}\right)=$ 0.120 the SPS1a' scenario corresponds to $m_{\tilde{g}}=610 \mathrm{GeV}, m_{\tilde{q}}=560 \mathrm{GeV}, \sin \left(2 \theta_{\tilde{t}}\right)=0.932$ and stop masses of $m_{\tilde{t}_{1}}=367 \mathrm{GeV}$ and $m_{\tilde{t}_{2}}=590 \mathrm{GeV}$ [54]. However, in order to focus on the mass dependence of the cross section and the NLO+NLL corrections, we vary the mass of the stop while keeping the other SUSY parameters fixed. As shown in ref. [3] and discussed in more detail in the appendix, the dependence of the cross section on the additional SUSY parameters is small, justifying this procedure. Note that the numerical results presented for stop production also apply to sbottom production when the same input parameters are adopted. In the appendix we show that the impact of bottom-quark induced contributions to sbottom hadroproduction is negligible and present benchmark predictions for the sbottom cross section.

\subsection{Results for the total cross section}

Let us first discuss the scale dependence of the SUSY-QCD total cross-section prediction. Figure 2 shows the scale dependence in LO, NLO and NLO+NLL for $\tilde{t}_{1} \overline{\tilde{t}}_{1}$ production, using $m_{\tilde{t}_{1}}=200 \mathrm{GeV}$ and $500 \mathrm{GeV}$ at the Tevatron and LHC, respectively. Here and in the following, we present results for the LHC operating at $7 \mathrm{TeV}$ and at $14 \mathrm{TeV}$ centre-of-mass energy. Note that the LO predictions are obtained with LO pdfs and the corresponding LO values for $\alpha_{\mathrm{s}}$ [48]. The renormalization and factorization scales are identified and varied around the central scale $\mu_{0}=m_{\tilde{t}_{1}}$ from $\mu=\mu_{0} / 10$ up to $\mu=5 \mu_{0}$. We observe the usual strong reduction of the scale dependence when going from LO to NLO. A further significant improvement is obtained when the resummation of threshold logarithms is included, in particular for stop production at the Tevatron and at the LHC running at $7 \mathrm{TeV}$.

Near the central scale $\mu=m_{\tilde{t}_{1}}$ the cross section is enhanced by the SUSY-QCD corrections at NLO and NLO+NLL. The size of the $K$-factors $K_{\mathrm{NLO}} \equiv \sigma_{\mathrm{NLO}} / \sigma_{\mathrm{LO}}$ and $K_{\mathrm{NLL}} \equiv \sigma_{\mathrm{NLO}+\mathrm{NLL}} / \sigma_{\mathrm{NLO}}$ strongly depends on the stop mass and the collider, as is shown in figure 3. At the Tevatron, where the cross section is dominated by $q \bar{q}$-annihilation for large stop masses, the NLO $K$-factor is moderate and ranges from roughly 1.2 to 1.03 for stop masses in the range between 100 and $300 \mathrm{GeV}$. A further enhancement by up to $7 \%$ is found for large stop masses when the NLL resummation is included.

At the LHC, the $g g$ initial state is dominant and the QCD corrections are in general larger. For $\tilde{t}_{1} \overline{\tilde{t}}_{1}$ and $\tilde{t}_{2} \overline{\tilde{t}}_{2}$ production at the $\mathrm{LHC}$ we consider the mass ranges $100 \mathrm{GeV} \leq$ $m_{\tilde{t}_{1}} \leq 550 \mathrm{GeV}$ (lower horizontal axis) and $550 \mathrm{GeV} \leq m_{\tilde{t}_{2}} \leq 1 \mathrm{TeV}$ (upper horizontal axis). At $7 \mathrm{TeV}$ we find NLO corrections ranging from about $40 \%$ at the lower end of the 


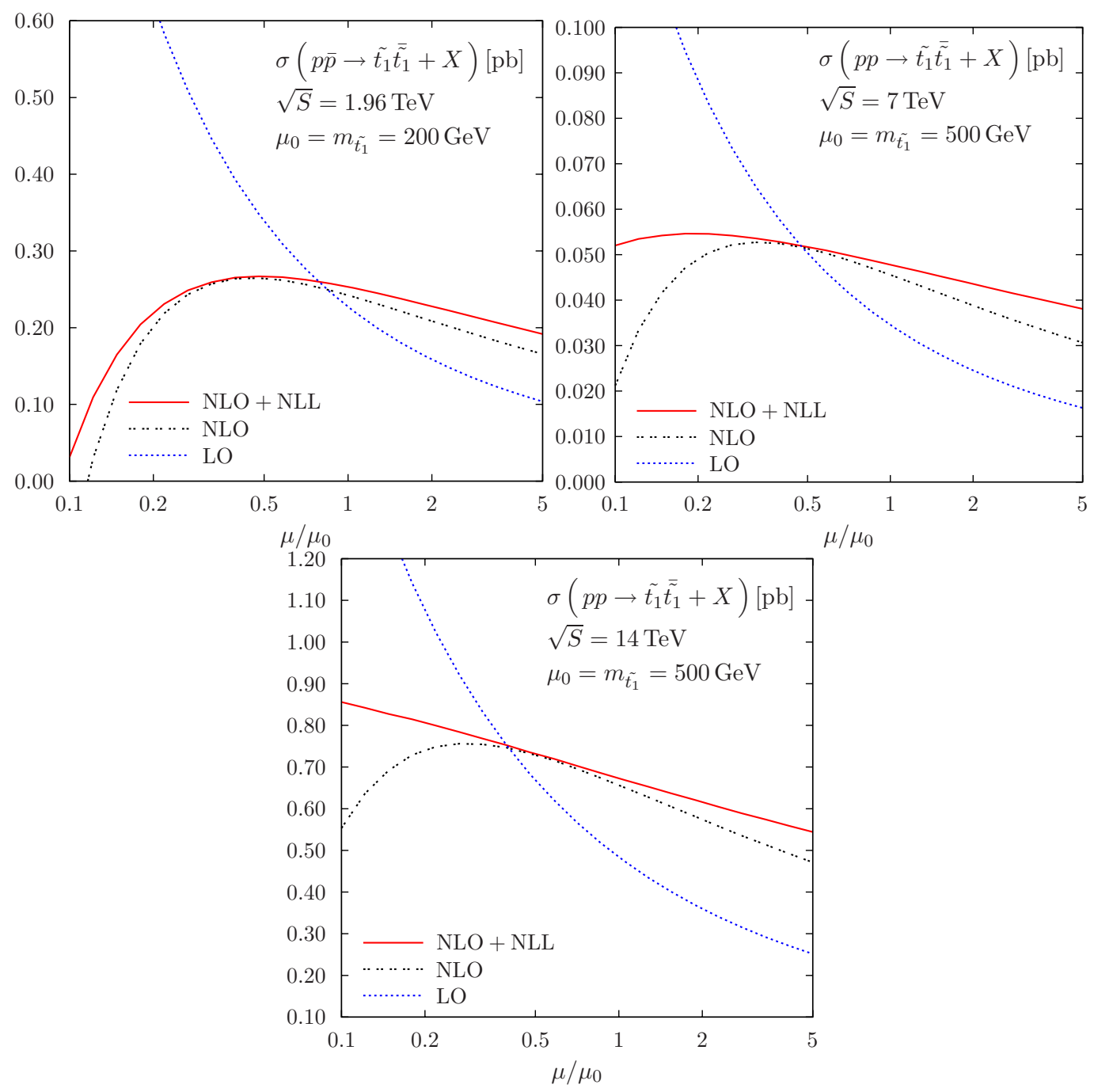

Figure 2. Scale dependence of the LO, NLO and NLO+NLL cross section for stop-antistop production at the Tevatron and the LHC.

stop mass range to about $20 \%$ for stop masses near $1 \mathrm{TeV}$. The NLL resummation leads to a further increase of the cross-section prediction of approximately $10 \%$ for stop masses in the TeV-range. At $14 \mathrm{TeV}$ centre-of-mass energy the NLO corrections to stop production are significant and increase the LO cross section by around $35 \%$ for moderate $\tilde{t}_{1}$ masses and by up to $40 \%$ for $\tilde{t}_{2}$ with $m_{\tilde{t}_{2}} \approx 600 \mathrm{GeV}$, while the impact of the NLL resummation is modest with at most $5 \%$ further increase for stop masses in the TeV-range. The singularities at the stop-decay threshold $m_{\tilde{t}}=m_{t}+m_{\tilde{g}}=782.5 \mathrm{GeV}$ originate from the stop wave-function renormalization. They are an unphysical artefact of the on-shell approach of ref. [3] and could be removed by taking into account the finite widths of the unstable stops. Note that the NLO cross sections for $\tilde{t}_{1} \overline{\tilde{t}}_{1}$ and $\tilde{t}_{2} \overline{\tilde{t}}_{2}$ production are not identical, even if the masses are taken equal. The reason for this is that the stop mixing angle contributes in different ways to both reactions at NLO, as discussed in section 2. Furthermore, while we vary the mass of the stop particle that appears in the final state, the mass of the other stop, 


\begin{tabular}{|c|c|c|c||c|c|c|}
\hline \multicolumn{7}{|c|}{$p \bar{p} \rightarrow \tilde{t_{1}} \tilde{t_{1}}$ at $\sqrt{S}=1.96 \mathrm{TeV}$} \\
\hline & \multicolumn{3}{|c||}{ MSTW2008 } & \multicolumn{3}{c|}{ CTEQ6.6M } \\
\hline$m_{\tilde{t_{1}}}[\mathrm{GeV}]$ & 100 & 200 & 300 & 100 & 200 & 300 \\
\hline$\left(\sigma \pm \Delta \sigma_{\mu}\right)_{\mathrm{LO}}[\mathrm{pb}]$ & $12.6_{-3.9}^{+6.4}$ & $0.227_{-0.068}^{+0.112}$ & $\left(1.12_{-0.35}^{+0.57}\right) \times 10^{-2}$ & $10.3_{-2.9}^{+4.3}$ & $0.210_{-0.059}^{+0.091}$ & $\left(1.20_{-0.33}^{+0.51}\right) \times 10^{-2}$ \\
\hline$\left(\sigma \pm \Delta \sigma_{\mu}\right)_{\mathrm{NLO}}[\mathrm{pb}]$ & $15.3_{-2.4}^{+2.0}$ & $0.242_{-0.034}^{+0.022}$ & $\left(1.15_{-0.17}^{+0.12}\right) \times 10^{-2}$ & $14.7_{-2.2}^{+1.8}$ & $0.249_{-0.034}^{+0.022}$ & $\left(1.23_{-0.18}^{+0.13}\right) \times 10^{-2}$ \\
\hline$\left(\sigma \pm \Delta \sigma_{\mu}\right)_{\mathrm{NLO}+\mathrm{NLL}}[\mathrm{pb}]$ & $15.9_{-1.8}^{+1.6}$ & $0.253_{-0.025}^{+0.014}$ & $\left(1.24_{-0.13}^{+0.07}\right) \times 10^{-2}$ & $15.1_{-1.6}^{+1.4}$ & $0.260_{-0.025}^{+0.014}$ & $\left(1.31_{-0.14}^{+0.08}\right) \times 10^{-2}$ \\
\hline$\Delta \mathrm{pdf}$ & \pm 6.6 & \pm 5.3 & \pm 5.3 & \pm 11 & \pm 11 & \pm 11 \\
\hline $\mathrm{K}_{\mathrm{NLO}}[\%]$ & 1.22 & 1.07 & 1.03 & 1.43 & 1.19 & 1.10 \\
\hline $\mathrm{K}_{\mathrm{NLL}}$ & 1.03 & 1.05 & 1.07 & 1.03 & 1.04 & 1.07 \\
\hline
\end{tabular}

Table 1. The LO, NLO and NLO+NLL cross sections for stop-antistop production at the Tevatron $(\sqrt{S}=1.96 \mathrm{TeV})$, including errors due to scale variation $\left(\Delta \sigma_{\mu}\right)$ in the range $m_{\tilde{t}_{1}} / 2 \leq \mu \leq 2 m_{\tilde{t}_{1}}$. Results are shown for two pdf parametrizations (MSTW08 and CTEQ6) with the corresponding 90\% C.L. pdf error estimates.

which enters the loop corrections, is set to its SPS1a' value and thus differs for $\tilde{t}_{1}$ and $\tilde{t}_{2}$. However, numerically the difference between the two NLO total cross sections is moderate. The NLL resummation does not involve any SUSY parameters apart from the stop mass itself and thus affects the $\tilde{t}_{1} \overline{\tilde{t}}_{1}$ and $\tilde{t}_{2} \overline{\tilde{t}}_{2}$ cross sections in the same way. The NLL $K$-factors have a tiny SUSY-parameter dependence, which enters through the ratio $\sigma_{\mathrm{NLO}+\mathrm{NLL}} / \sigma_{\mathrm{NLO}}$.

Predictions for the LO, NLO, and NLO+NLL total cross sections are shown in figure 4 and tables $1-3$ for $\tilde{t}_{1} \overline{\tilde{t}}_{1}$ production at the Tevatron and $\tilde{t}_{1} \overline{\tilde{t}}_{1} / \tilde{t}_{2} \overline{\tilde{t}}_{2}$ production at the LHC with $7 \mathrm{TeV}$ and $14 \mathrm{TeV}$ centre-of-mass energy. In fact, the cross sections for $\tilde{t}_{1} \overline{\tilde{t}}_{1}$ and $\tilde{t}_{2} \overline{\tilde{t}}_{2}$ production at equal masses are indistinguishable on the scale of figure 4 . We thus refrain from showing additional plots for $\tilde{t}_{2} \overline{\tilde{t}}_{2}$ production. The results shown in figure 4 represent the state-of-the-art SUSY-QCD predictions at NLO+NLL accuracy. The error bands include the NLO+NLL scale variation in the range $m_{\tilde{t}_{1}} / 2 \leq \mu \leq 2 m_{\tilde{t}_{1}}$ as well as the NLO pdf uncertainty, added in quadrature. The pdf uncertainty is obtained with the help of the $90 \%$ C.L. MSTW error pdfs [49]. More detailed information is available in tables 1-3.

In table 1 we present results for $\tilde{t}_{1} \bar{t}_{1}$ production at the Tevatron. As discussed before, we observe an increase of the cross-section prediction near the central scale when going from LO to NLO and a further enhancement when NLL threshold resummation is included. The scale dependence in the range $m_{\tilde{t}_{1}} / 2 \leq \mu \leq 2 m_{\tilde{t}_{1}}$ is reduced from about $\pm 50 \%$ at LO to about $\pm 10 \%$ at NLO+NLL. The estimated pdf uncertainty is approximately $5 \%$. We also present cross-section predictions obtained with the CTEQ6 pdf set [55] and an estimate of the corresponding pdf error. The difference between the MSTW and CTEQ results is particularly pronounced at large stop masses, $m_{\tilde{t}_{1}} \approx 300 \mathrm{GeV}$, where the cross sections obtained with CTEQ pdfs are about 7\% larger than the ones obtained with MSTW pdfs. We observe that the CTEQ pdf error estimate of about $10 \%$ is roughly twice as large as that of MSTW.

Results for $\tilde{t}_{1} \overline{\tilde{t}}_{1}$ and $\tilde{t}_{2} \overline{\tilde{t}}_{2}$ production at the LHC with $7 \mathrm{TeV}$ are collected in table 2 . Here the impact of the SUSY-QCD corrections at NLO is large, with the NLL resummation adding a further enhancement of up to $10 \%$. The scale uncertainty of the NLO+NLL prediction is reduced to a level of about $10 \%$. Unfortunately the pdf error is sizeable, 

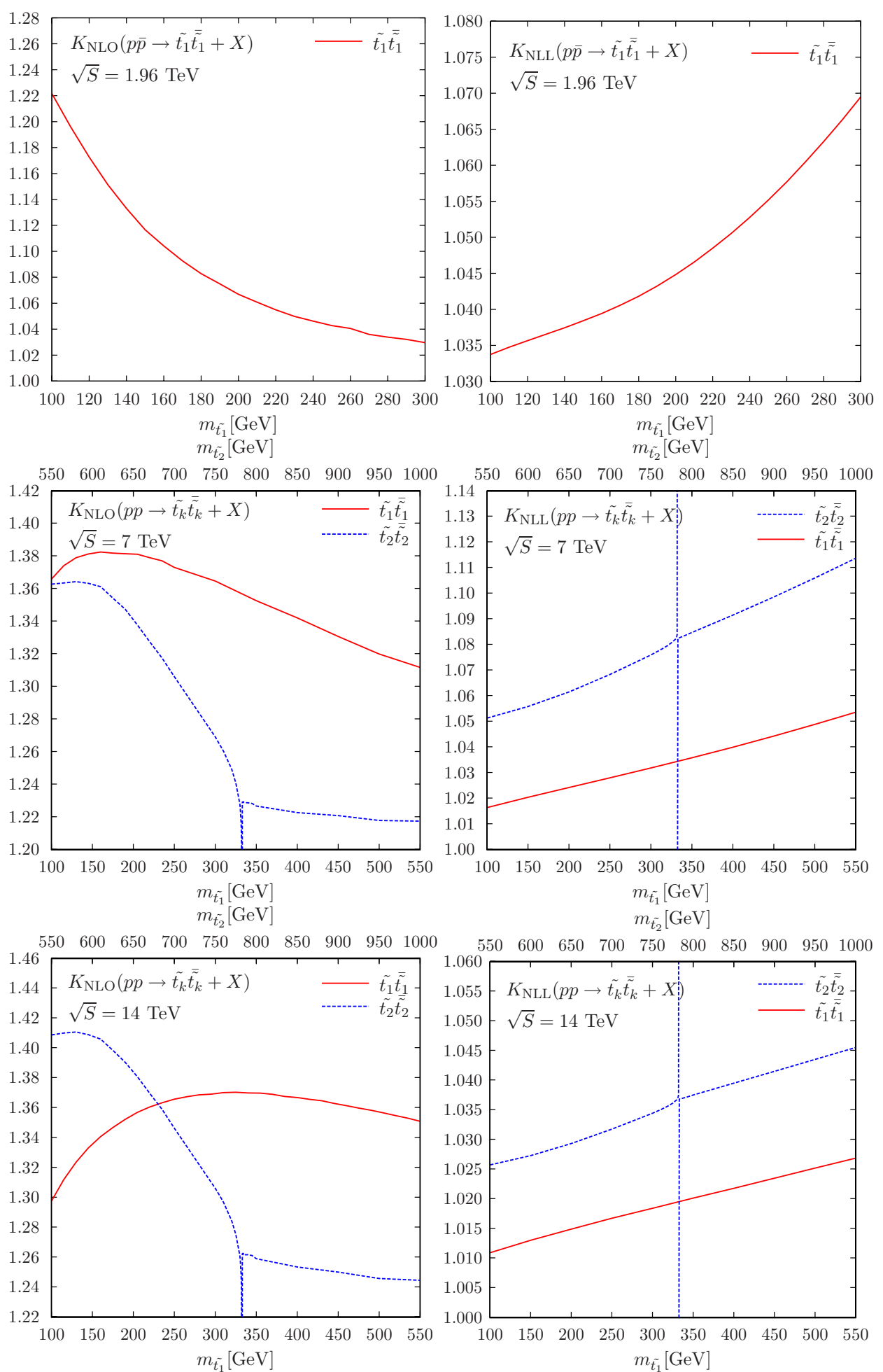

Figure 3. The NLO and NLL $K$-factors for stop-antistop production at the Tevatron and the LHC as a function of the stop mass. The scale has been set to the stop mass, i.e. $\mu=m_{\tilde{t}_{k}}$. 


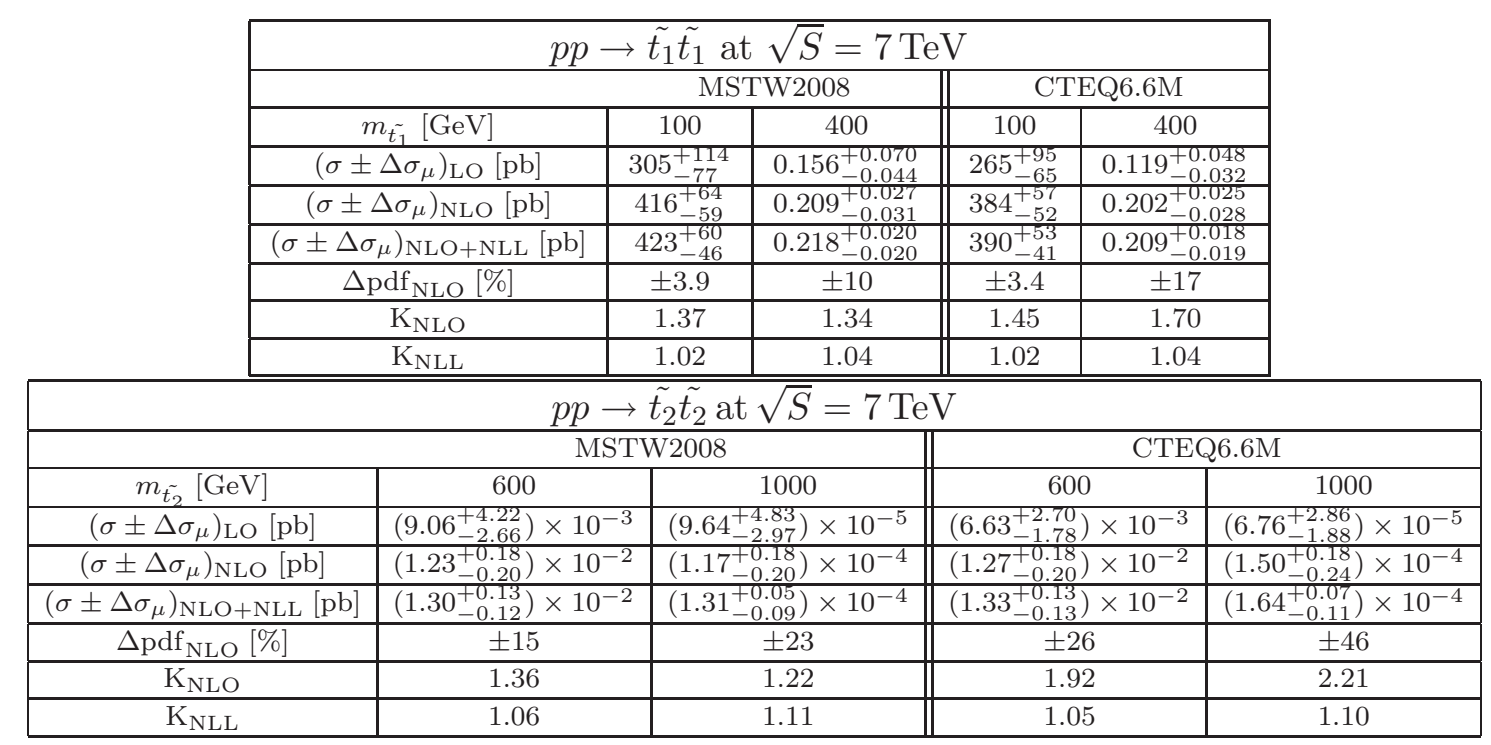

Table 2. The LO, NLO and NLO+NLL cross sections for stop-antistop production at the LHC $(\sqrt{S}=7 \mathrm{TeV})$, including errors due to scale variation $\left(\Delta \sigma_{\mu}\right)$ in the range $m_{\tilde{t}} / 2 \leq \mu \leq 2 m_{\tilde{t}}$. Results are shown for two pdf parametrizations (MSTW08 and CTEQ6) with the corresponding 90\% C.L. pdf error estimates.

in particular at stop masses in the TeV-region, where we find a pdf error of about $20 \%$ from MSTW. Also the difference between MSTW and CTEQ is significant for large stop masses, with a $25 \%$ increase in the prediction for stop masses near $1 \mathrm{TeV}$ when going from MSTW to CTEQ pdfs. As before, we find a pdf error from the CTEQ analysis that is about twice as large as that of MSTW and reaches $45 \%$ for $m_{\tilde{t}} \approx 1 \mathrm{TeV}$. Of course, the large pdf uncertainty is not a specific feature of stop production. It rather generically affects predictions for $\mathrm{TeV}$-scale particle production at the $\mathrm{LHC}$ with $7 \mathrm{TeV}$ centre-ofmass energy, since these predictions are particularly sensitive to the gluon pdf at large $x$ (see e.g. ref. [56]). The conclusion therefore is that more accurate pdf determinations are needed in order to allow for a precise prediction of heavy-particle production during the initial phase of the LHC at $7 \mathrm{TeV}$ centre-of-mass energy.

Going from $7 \mathrm{TeV}$ to $14 \mathrm{TeV}$ at the LHC, we observe in table 3 a significant increase in the predicted cross section of about a factor of 4 for stop masses around $100 \mathrm{GeV}$ and up to a factor of about 60 for masses in the TeV-region and MSTW pdfs. Just like at $7 \mathrm{TeV}$, the scale uncertainty of the NLO+NLL prediction is down to a level of about $10 \%$. The pdf uncertainty is more moderate than at $7 \mathrm{TeV}$, ranging from $3 \%$ at small masses to about $10 \%$ at large stop masses for MSTW, and correspondingly from $3 \%$ to $20 \%$ for CTEQ.

\subsection{Results for the transverse-momentum distribution}

Let us now turn to the discussion of the transverse-momentum distributions. Figure 5 shows a comparison between the LO, NLO and NLO+NLL distributions normalized to unity. We use normalized distributions in order to be able to directly read off the NLOand NLL-induced changes in the shape of the distribution. As for the previous results, we 


\begin{tabular}{|c|c|c|c|c|c|c|}
\hline \multicolumn{7}{|c|}{$p p \rightarrow \tilde{t_{1}} \tilde{t}_{1}$ at $\sqrt{S}=14 \mathrm{TeV}$} \\
\hline \multicolumn{4}{|c|}{ MSTW2008 } & \multicolumn{3}{|c|}{ CTEQ6.6M } \\
\hline$m_{\tilde{t_{1}}}[\mathrm{GeV}]$ & \multicolumn{2}{|c|}{100} & 400 & \multicolumn{2}{|l|}{100} & 400 \\
\hline$\left(\sigma \pm \Delta \sigma_{\mu}\right)_{\mathrm{LO}}[\mathrm{pb}]$ & \multicolumn{2}{|c|}{$\left(1.35_{-0.29}^{+0.41}\right) \times 10^{3}$} & $1.67_{-0.42}^{+0.62}$ & \multicolumn{2}{|c|}{$\left(1.22_{-0.26}^{+0.35}\right) \times 10^{3}$} & \begin{tabular}{|l}
$1.40_{-0.34}^{+0.49}$ \\
\end{tabular} \\
\hline$\left(\sigma \pm \Delta \sigma_{\mu}\right)_{\mathrm{NLO}}[\mathrm{pb}]$ & \multicolumn{2}{|c|}{$\left(1.75_{-0.22}^{+0.26}\right) \times 10^{3}$} & $2.29_{-0.29}^{+0.25}$ & \multicolumn{2}{|c|}{$\left(1.63_{-0.19}^{+0.23}\right) \times 10^{3}$} & $2.14_{-0.26}^{+0.24}$ \\
\hline$\left(\sigma \pm \Delta \sigma_{\mu}\right)_{\mathrm{NLO}+\mathrm{NLL}}[\mathrm{pb}]$ & \multicolumn{2}{|c|}{$\left(1.77_{-0.17}^{+0.24}\right) \times 10^{3}$} & $2.34_{-0.21}^{+0.21}$ & \multicolumn{2}{|c|}{$\left(1.65_{-0.16}^{+0.22}\right) \times 10^{3}$} & $2.19_{-0.19}^{+0.20}$ \\
\hline$\Delta \mathrm{pdf}_{\mathrm{NLO}}[\%]$ & \multicolumn{2}{|c|}{ \pm 2.8} & \pm 6.2 & \multicolumn{2}{|c|}{ \pm 2.6} & \pm 8.6 \\
\hline \multirow{2}{*}{$\begin{array}{l}\mathrm{K}_{\mathrm{NLO}} \\
\mathrm{K}_{\mathrm{NLL}}\end{array}$} & \multicolumn{2}{|c|}{1.30} & 1.37 & \multicolumn{2}{|l|}{1.34} & 1.53 \\
\hline & \multicolumn{2}{|c|}{1.01} & 1.02 & \multicolumn{2}{|l|}{1.01} & 1.02 \\
\hline \multicolumn{7}{|c|}{$p p \rightarrow \tilde{t_{2}} \tilde{t_{2}}$ at $\sqrt{S}=14 \mathrm{TeV}$} \\
\hline & \multicolumn{3}{|c|}{ MSTW2008 } & \multicolumn{3}{|c|}{ CTEQ6.6M } \\
\hline$\overline{m_{\tilde{t_{2}}}}[\mathrm{GeV}]$ & \multirow{2}{*}{$\frac{600}{0.167_{-0.043}^{+0.065}}$} & \multicolumn{2}{|c|}{1000} & \multicolumn{3}{|c|}{\begin{tabular}{l|l} 
& 1000
\end{tabular}} \\
\hline$\left(\sigma \pm \Delta \sigma_{\mu}\right)_{\mathrm{LO}}[\mathrm{pb}]$ & & \multicolumn{2}{|c|}{$\left(6.13_{-1.65}^{+2.51}\right) \times 10^{-3}$} & $0.135_{-0.033}^{+0.048}$ & \multicolumn{2}{|c|}{$\left(4.71_{-1.17}^{+1.72}\right) \times 10^{-3}$} \\
\hline$\left(\sigma \pm \Delta \sigma_{\mu}\right)_{\mathrm{NLO}}[\mathrm{pb}]$ & & \multicolumn{2}{|c|}{$\left(7.63_{-0.92}^{+0.65}\right) \times 10^{-3}$} & $0.225_{-0.029}^{+0.027}$ & \multicolumn{2}{|c|}{$\left(7.65_{-0.90}^{+0.62}\right) \times 10^{-3}$} \\
\hline$\left(\sigma \pm \Delta \sigma_{\mu}\right)_{\mathrm{NLO}+\mathrm{NLL}}[\mathrm{pb}]$ & $\frac{0.235_{-0.031}^{+0.030}}{0.242_{-0.022}^{+0.0244}}$ & \multicolumn{2}{|c|}{$\left(7.98_{-0.51}^{+0.36}\right) \times 10^{-3}$} & $0.230_{-0.020}^{+0.022}$ & $(7.97$ & $\left.\begin{array}{l}+0.35 \\
-0.50\end{array}\right) \times 10^{-3}$ \\
\hline$\Delta \mathrm{pdf}_{\mathrm{NLO}}[\%]$ & \pm 8.3 & & 12 & \pm 13 & & \pm 21 \\
\hline $\mathrm{K}_{\mathrm{NLO}}$ & 1.41 & & 24 & 1.66 & & 1.62 \\
\hline $\mathrm{K}_{\mathrm{NLL}}$ & 1.03 & & 05 & 1.03 & & 1.04 \\
\hline
\end{tabular}

Table 3. The LO, NLO and NLO+NLL cross sections for stop-antistop production at the LHC $(\sqrt{S}=14 \mathrm{TeV})$, including errors due to scale variation $\left(\Delta \sigma_{\mu}\right)$ in the range $m_{\tilde{t}} / 2 \leq \mu \leq 2 m_{\tilde{t}}$. Results are shown for two pdf parametrizations (MSTW08 and CTEQ6) with the corresponding 90\% C.L. pdf error estimates.

have used the stop mass as the central scale, $\mu=m$, in figure 5 . This is a possible choice as we do not consider regions where $p_{T} \gg m$ and where a $p_{T}$-dependent scale would have been mandatory. As already observed in ref. [57], the transverse momentum carried away by hard gluon radiation in higher orders softens the NLO transverse-momentum distribution with respect to the $\mathrm{LO}$ distribution. This effect is particularly visible at the Tevatron and at the LHC with $7 \mathrm{TeV}$ centre-of-mass energy. The NLL soft-gluon resummation, on the other hand, does not affect the shape of the distribution significantly. To elucidate the impact of the higher-order corrections more clearly, we display the transverse-momentum dependence of the NLO and NLL $K$-factors in figure 6 , this time using the transverse mass $m_{T}=\sqrt{m^{2}+p_{T}^{2}}$ as the scale. The significant softening of the transverse-momentum distribution at NLO at the Tevatron and the $7 \mathrm{TeV}$ LHC is reflected in the variation of the $K$-factor, with $K_{\mathrm{NLO}}$ dropping from roughly 1.8 at small $p_{T}$ to a value near one at $p_{T} \approx 2 m$. In comparison, the impact of the NLL resummation is small. A similar behaviour, albeit less pronounced, is observed at the LHC with $14 \mathrm{TeV}$ centre-of-mass energy. It would be interesting to see if using NLO+NLL transverse-momentum distributions would affect the experimental analyses, which so far have been based on LO Monte Carlo predictions. In this context we recall that the shape of the stop rapidity distribution is not changed significantly by higher-order corrections, see ref. [57]. 


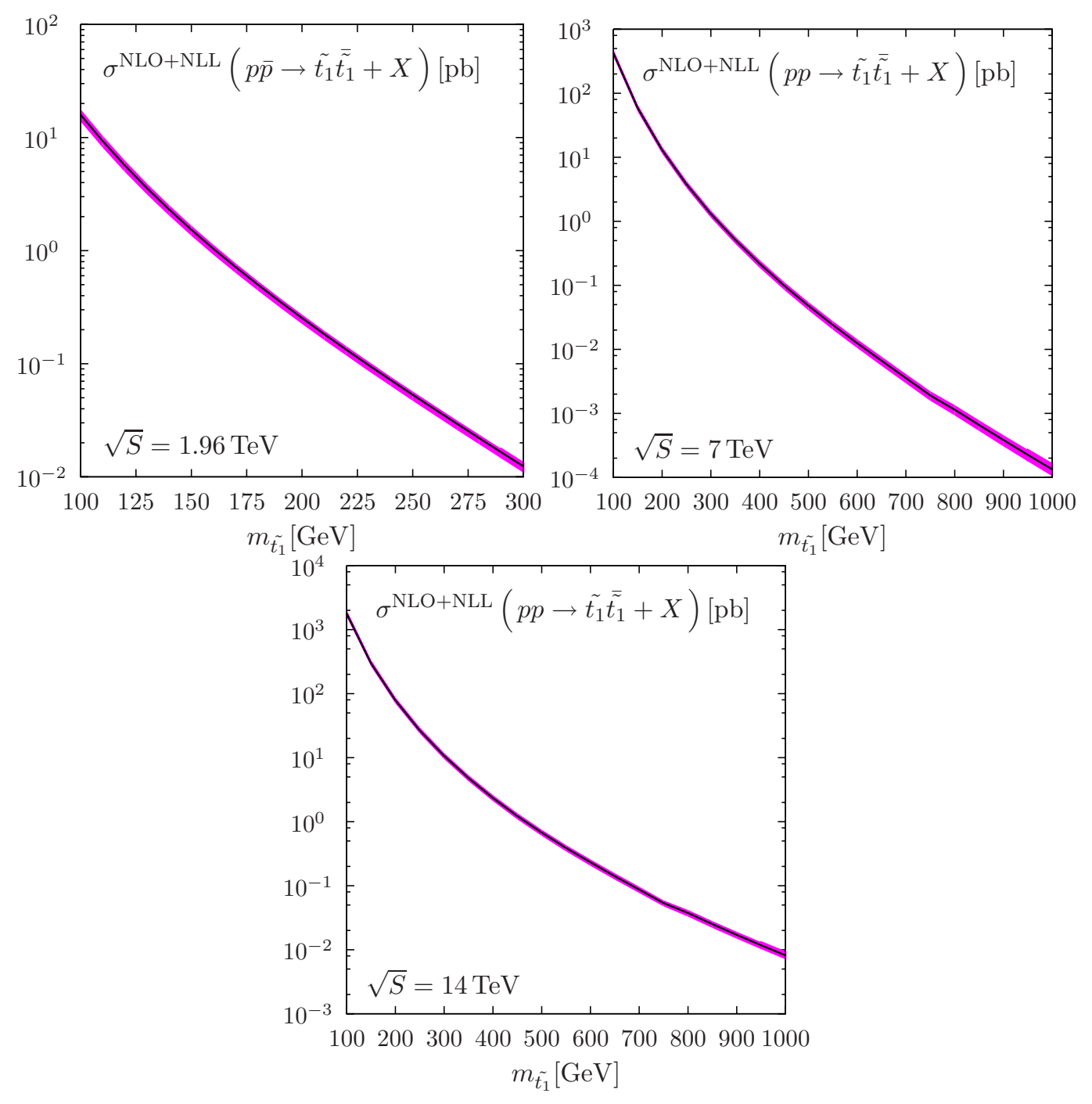

Figure 4. Total NLO+NLL stop-pair cross section at the Tevatron and the LHC as a function of the stop mass. The error band corresponds to the scale and pdf uncertainty of the prediction, added in quadrature.

\section{Conclusions}

In this paper we have performed the NLL threshold resummation for stop and sbottom hadroproduction, considering both the inclusive cross sections and the transversemomentum distributions. As the lighter stop and sbottom mass eigenstates are generally predicted to be the lightest strongly interacting SUSY particles, the search for these particles plays a special role in the quest to find signals of supersymmetry at hadron colliders.

Results have been given for the Tevatron and for the LHC running at both $7 \mathrm{TeV}$ and $14 \mathrm{TeV}$ centre-of-mass energy. Compared to the NLO predictions for the total cross section, the NLL corrections lead to a significant reduction of the scale dependence and increase the cross section by up to $10 \%$ for masses in the $\mathrm{TeV}$ range if the renormalization and factorization scales are chosen close to the mass of the final-state particles. We have also 


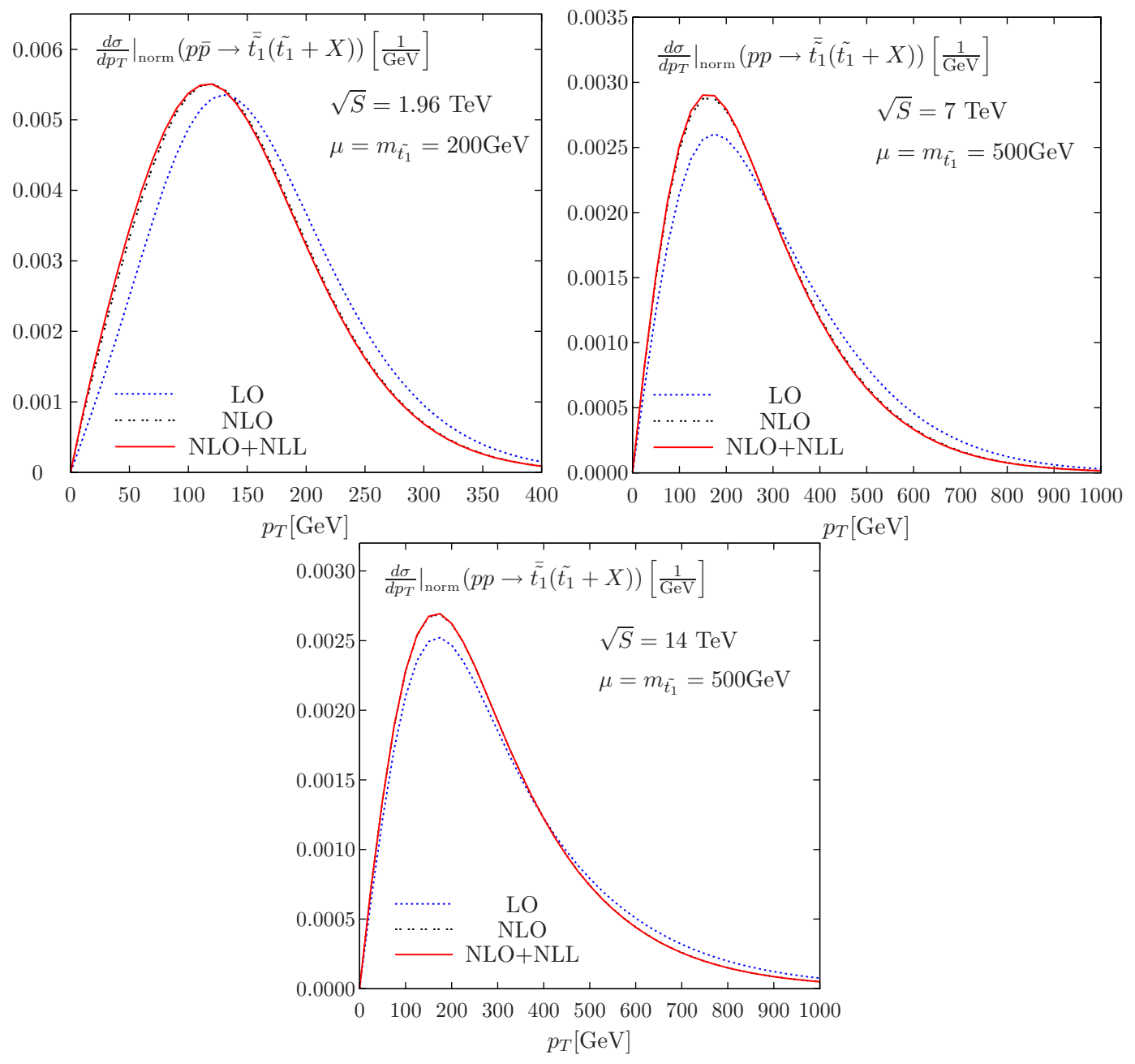

Figure 5. Normalized LO, NLO and NLO+NLL transverse-momentum distributions for stopantistop production at the Tevatron and the LHC for $\mu=m_{\tilde{t}_{1}}$.

studied the SUSY parameter dependence of the stop and sbottom cross sections and find small variations of at most $2 \%$. The size of bottom-induced contributions to sbottom pair production is negligible numerically so that predictions obtained for stop-pair production also apply to sbottom-pair production when the same input parameters are adopted.

Since $p_{T}$ cuts are used extensively in experimental analyses, which at present are based on LO Monte Carlo simulations, it is important to investigate how the NLO+NLL matched corrections affect the transverse-momentum distributions. We find that the NLO+NLL corrections can change the shape of the $p_{T}$ distribution considerably and thus generally cannot be taken into account by using a simple $K$-factor.

The NLO+NLL matched cross sections and $p_{T}$ distributions presented in this paper constitute the state-of-the-art QCD predictions for stop and sbottom production and can be employed to improve current and future searches at the Tevatron and LHC. 

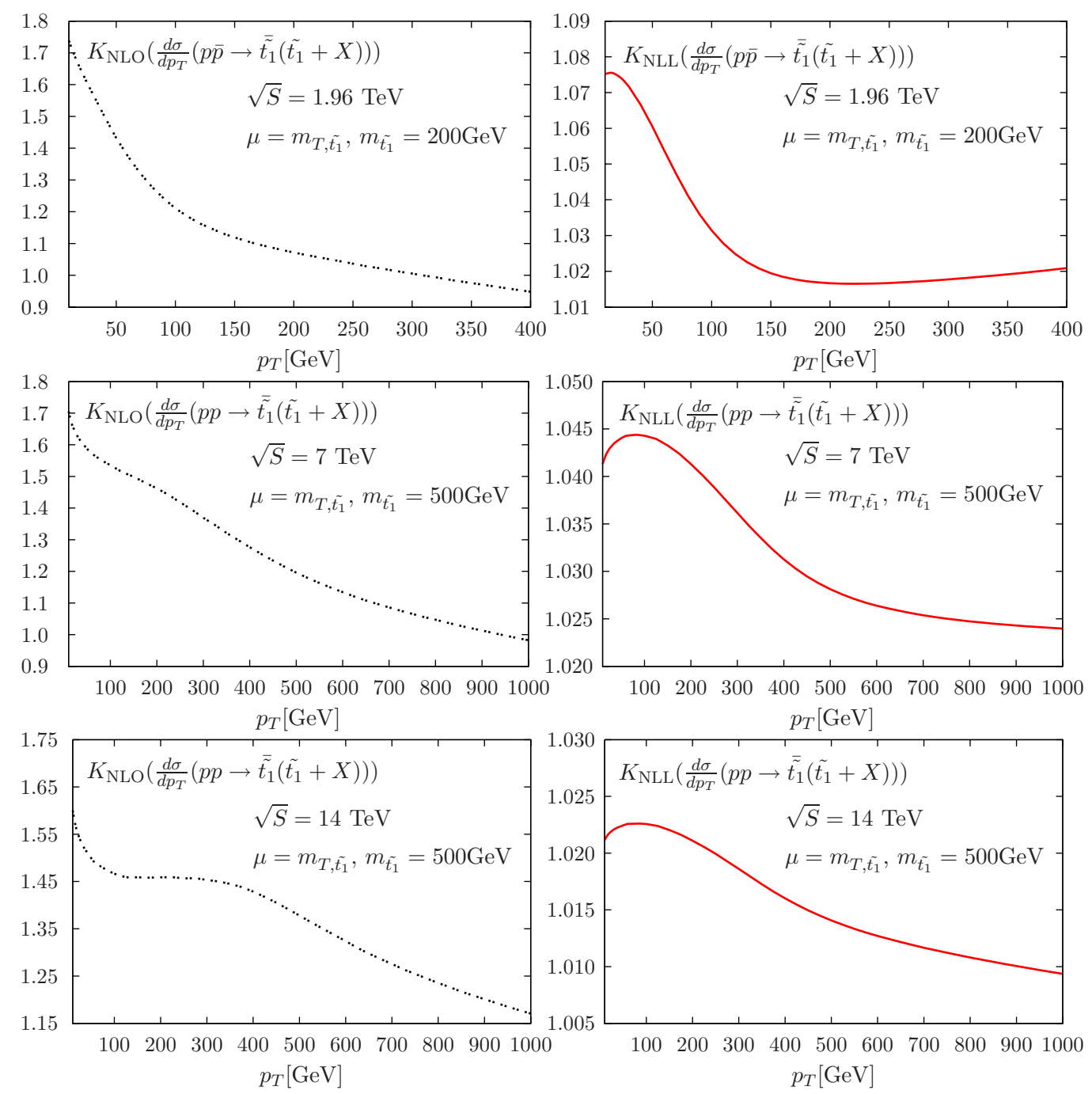

Figure 6. Transverse-momentum dependence of the NLO and NLL $K$-factors for stop-antistop production at the Tevatron and the LHC for $\mu=m_{T, \tilde{t}_{1}}$.

\section{Acknowledgments}

We would like to thank Sven Moch, James Stirling, Robert Thorne and Andreas Vogt for valuable discussions and the MSTW collaboration for providing us with parton distribution functions with improved accuracy in the evolution at large $x$. This work has been supported in part by the Helmholtz Alliance "Physics at the Terascale", the DFG Graduiertenkolleg "Elementary Particle Physics at the TeV Scale", the Foundation for Fundamental Research of Matter (FOM), the National Organization for Scientific Research (NWO), the DFG SFB/TR9 "Computational Particle Physics", and the European Community's Marie-Curie Research Training Network under contract MRTN-CT-2006-035505 "Tools and Precision Calculations for Physics Discoveries at Colliders". SB would like to thank the Nikhef Theory Group, and MK would like to thank the Institute for High Energy Physics (IFAE) at the Universitat Autónoma de Barcelona and the CERN TH 


\begin{tabular}{|c|c||c|c||c|c|}
\hline \multicolumn{7}{|c|}{ SPS1a' } \\
\hline$m_{\tilde{t}_{1}}$ & $367 \mathrm{GeV}$ & $m_{\tilde{b}_{1}}$ & $509 \mathrm{GeV}$ & $m_{\tilde{q}}$ & $560 \mathrm{GeV}$ \\
$m_{\tilde{t}_{2}}$ & $590 \mathrm{GeV}$ & $m_{\tilde{b}_{2}}$ & $549 \mathrm{GeV}$ & $m_{\tilde{g}}$ & $610 \mathrm{GeV}$ \\
$\sin 2 \theta_{\tilde{t}}$ & 0.932 & $\sin 2 \theta_{\tilde{b}}$ & 0.652 & & \\
\hline
\end{tabular}

Table 4. Masses and mixings for the SPS1a' benchmark scenario [52] obtained using SPheno [54] with the Standard Model input parameters $m_{t}=172.5 \mathrm{GeV}$ and $\alpha_{\mathrm{s}}\left(M_{Z}\right)=0.120$.

\begin{tabular}{|c|c|c|}
\hline \multirow{2}{*}{ SPS1a' } & \multicolumn{2}{|c|}{$\sigma_{\mathrm{NLO}+\mathrm{NLL}}[\mathrm{pb}]$} \\
\cline { 2 - 3 } & LHC @ 7 TeV & LHC @ $14 \mathrm{TeV}$ \\
\hline$\tilde{t}_{1} \tilde{t}_{1}$ & 0.379 & 3.71 \\
$\tilde{t}_{2} \tilde{\hat{t}}_{2}$ & $1.48 \times 10^{-2}$ & 0.268 \\
$\tilde{b}_{1} \tilde{b}_{1}$ & $4.23 \times 10^{-2}$ & 0.611 \\
$\tilde{b}_{2} \tilde{\tilde{b}}_{2}$ & $2.51 \times 10^{-2}$ & 0.405 \\
\hline
\end{tabular}

Table 5. NLO+NLL SUSY-QCD cross sections for stop and sbottom pair production at the LHC for the SPS1a' benchmark scenario. The MSTW pdfs have been adopted and the scale has been set to the mass of the particles produced.

unit for their hospitality. AK acknowledges the hospitality of the Institute of Theoretical Physics at the University of Warsaw.

\section{A SUSY parameter dependence of stop and sbottom cross sections}

In this appendix we shall investigate the dependence of the NLO+NLL stop and sbottom cross-section predictions on the supersymmetric model parameters that enter beyond LO, i.e. the mixing angle and the masses of the light-flavour squarks and the gluino. In the case of sbottom production we shall in addition quantify the impact of the bottom-quarkinduced reaction channels $b \bar{b} \rightarrow \tilde{b}_{k} \overline{\tilde{b}}_{k}$ and $b b / \bar{b} \bar{b} \rightarrow \tilde{b}_{k} \tilde{b}_{k} / \overline{\tilde{b}}_{k} \overline{\tilde{b}}_{k}$, involving contributions from $t$-channel gluino exchange. It will be demonstrated that the contributions of these partonic reaction channels are strongly suppressed numerically. Thus, for all practical purposes, cross-section predictions obtained for stop-pair production also apply to sbottom-pair production when the same input parameters are adopted.

As in the main body of the paper we choose the SPS1a' benchmark scenario [52] as our default. The SPS1a' masses and mixings relevant for stop and sbottom hadroproduction are collected in table 4 . Note that the stop and sbottom masses predicted by the SPS1a' and other commonly used benchmark scenarios are beyond the reach of the Tevatron searches, as the corresponding production cross sections are too small. The SPS1a' NLO+NLL cross sections for stop and sbottom production at the LHC are collected in table 5. From the cross-section predictions one can conclude that only the lighter of the SPS1a' stop mass eigenstates might be detected during the initial phase of LHC data taking at $7 \mathrm{TeV}$ with $1 \mathrm{fb}^{-1}$ of integrated luminosity. Also a dedicated search for sbottom production in SPS1a'-like scenarios will only be possible with higher LHC energies.

Therefore, to address the SUSY-parameter dependence and to study the impact of bottom-quark-induced sbottom-pair production, we consider two different scenarios that 


\begin{tabular}{|r|c|c||c|c||c|c|}
\hline \multicolumn{9}{|c|}{$\sigma\left(p p / p \bar{p} \rightarrow \tilde{t}_{1} \tilde{t}_{1}\right)[\mathrm{pb}]$} \\
\hline & \multicolumn{2}{|c||}{ Tevatron $(1.96 \mathrm{TeV})$} & \multicolumn{2}{|c||}{ LHC $9 \mathrm{TeV}$} & \multicolumn{2}{c|}{ LHC @ $14 \mathrm{TeV}$} \\
\hline$m_{\tilde{t}_{1}}[\mathrm{GeV}]$ & 100 & 200 & 100 & 400 & 100 & 400 \\
\hline SPS1a' default & 15.9 & 0.253 & 423 & 0.218 & $1.77 \times 10^{3}$ & 2.34 \\
\hline $\sin 2 \theta_{\tilde{t}}=-1$ & 15.9 & 0.255 & 425 & 0.222 & $1.78 \times 10^{3}$ & 2.39 \\
0 & 15.9 & 0.254 & 423 & 0.219 & $1.77 \times 10^{3}$ & 2.36 \\
+1 & 15.9 & 0.253 & 423 & 0.218 & $1.77 \times 10^{3}$ & 2.33 \\
\hline$m_{\tilde{q}}=200 \mathrm{GeV}$ & 15.8 & 0.248 & 423 & 0.217 & $1.77 \times 10^{3}$ & 2.34 \\
$500 \mathrm{GeV}$ & 15.9 & 0.252 & 423 & 0.218 & $1.77 \times 10^{3}$ & 2.34 \\
$1000 \mathrm{GeV}$ & 15.9 & 0.255 & 423 & 0.219 & $1.77 \times 10^{3}$ & 2.34 \\
\hline$m_{\tilde{g}}=200 \mathrm{GeV}$ & 15.8 & 0.251 & 421 & 0.214 & $1.76 \times 10^{3}$ & 2.29 \\
$500 \mathrm{GeV}$ & 15.9 & 0.253 & 423 & 0.217 & $1.77 \times 10^{3}$ & 2.33 \\
$1000 \mathrm{GeV}$ & 15.9 & 0.254 & 424 & 0.219 & $1.77 \times 10^{3}$ & 2.34 \\
\hline
\end{tabular}

Table 6. The NLO+NLL cross sections for stop-antistop production at the Tevatron and the LHC. We compare the SPS1a' default input for the stop mixing angle and the light-flavour squark and gluino masses with various other choices for these SUSY parameters. Note that only one parameter is changed at a time, while the others are kept at their default values. The MSTW pdfs have been adopted and the scale has been set to the final-state stop mass.

\begin{tabular}{|r|c|c||c|c||c|c|}
\hline \multicolumn{9}{|c|}{$\sigma\left(p p / p \bar{p} \rightarrow \tilde{b}_{1} \tilde{b}_{1}\right)[\mathrm{pb}]$} \\
\hline$m_{\tilde{b}_{1}}[\mathrm{GeV}]$ & 100 & 200 & 100 & 400 & \multicolumn{2}{c|}{ LHC @ $14 \mathrm{TeV}$} \\
\hline SPS1a default & \multicolumn{1}{|c||}{ Tevatron $(1.96 \mathrm{TeV})$} & \multicolumn{2}{c|}{ LHC @ 7 TeV } & 100 & 400 \\
NLO+NLL & 15.9 & 0.253 & 423 & 0.218 & $1.77 \times 10^{3}$ & 2.34 \\
LO & 12.6 & 0.227 & 305 & 0.156 & $1.35 \times 10^{3}$ & 1.67 \\
LO $b \bar{b}$ channel only & $0.404 \times 10^{-2}$ & $0.330 \times 10^{-4}$ & 0.275 & $0.346 \times 10^{-3}$ & 1.40 & $0.564 \times 10^{-2}$ \\
\hline LO $b \bar{b}$ channel only & & & & & & \\
with $m_{\tilde{g}}=200 \mathrm{GeV}$ & $0.986 \times 10^{-2}$ & $0.870 \times 10^{-4}$ & 0.659 & $0.667 \times 10^{-3}$ & 3.35 & $0.111 \times 10^{-1}$ \\
$500 \mathrm{GeV}$ & $0.454 \times 10^{-2}$ & $0.399 \times 10^{-4}$ & 0.309 & $0.408 \times 10^{-3}$ & 1.58 & $0.665 \times 10^{-2}$ \\
$1000 \mathrm{GeV}$ & $0.335 \times 10^{-2}$ & $0.220 \times 10^{-4}$ & 0.227 & $0.220 \times 10^{-3}$ & 1.16 & $0.360 \times 10^{-2}$ \\
\hline
\end{tabular}

Table 7. The LO and NLO+NLL cross sections for sbottom-antisbottom production at the Tevatron and the LHC. We compare the default SPS1a' prediction for the stop-like contributions with the LO contributions induced by bottom-quark initial states. The MSTW pdfs have been adopted and the scale has been set to the final-state sbottom mass.

are within the reach of the Tevatron and the early LHC phase. As we did in the main body of the paper, we use stop and sbottom masses of $100(200) \mathrm{GeV}$ at the Tevatron and 100 (400) $\mathrm{GeV}$ at the LHC, respectively, and present results for various choices of the mixing angle and the light-flavour squark and gluino masses, see table 6. Note that the NLL resummation only depends on the final-state particle mass. The dependence on the other SUSY parameters enters exclusively through the NLO virtual corrections. The numbers listed in table 6 reveal that the dependence of the cross section on the mixing angle, the gluino mass, and the light-flavour squark masses is small indeed, with variations of at most $2 \%$ both at the Tevatron and at the LHC. 


\begin{tabular}{|r|c|c||c|c||c|c|}
\hline \multicolumn{9}{|c|}{$\sigma\left(p p / p \bar{p} \rightarrow \tilde{b}_{1} \tilde{b}_{1}\right)[\mathrm{pb}]$} \\
\hline$m_{\tilde{b}_{1}}[\mathrm{GeV}]$ & 100 & 200 & \multicolumn{2}{|c||}{ LHC @ $7 \mathrm{TeV}$} & \multicolumn{2}{c|}{ LHC @ $14 \mathrm{TeV}$} \\
& Tevatron $(1.96 \mathrm{TeV})$ & 100 & 400 & 100 & 400 \\
\hline SPS1a' default & $0.111 \times 10^{-2}$ & $0.188 \times 10^{-4}$ & $0.716 \times 10^{-1}$ & $0.205 \times 10^{-3}$ & 0.362 & $0.306 \times 10^{-2}$ \\
$m_{\tilde{g}}=200 \mathrm{GeV}$ & $0.568 \times 10^{-2}$ & $0.518 \times 10^{-4}$ & 0.335 & $0.242 \times 10^{-3}$ & 1.64 & $0.376 \times 10^{-2}$ \\
$500 \mathrm{GeV}$ & $0.157 \times 10^{-2}$ & $0.247 \times 10^{-4}$ & $0.994 \times 10^{-1}$ & $0.234 \times 10^{-3}$ & 0.500 & $0.349 \times 10^{-2}$ \\
$1000 \mathrm{GeV}$ & $0.447 \times 10^{-3}$ & $0.846 \times 10^{-5}$ & $0.297 \times 10^{-1}$ & $0.124 \times 10^{-3}$ & 0.153 & $0.187 \times 10^{-2}$ \\
\hline
\end{tabular}

Table 8. The LO cross sections for $\tilde{b}_{1} \tilde{b}_{1}$ production at the Tevatron and the LHC. The MSTW pdfs have been adopted and the scale has been set to the final-state sbottom mass.

Cross sections for $\tilde{b}_{1} \overline{\tilde{b}}_{1}$ production are collected in table 7 . We compare the LO bottomquark-induced contributions with the LO and NLO+NLL predictions based on the stop-like contributions that exclude bottom-quark initial states. Using the notation introduced in section 2 and $m_{-}^{2} \equiv m_{\tilde{g}}^{2}-m_{\tilde{b}_{1}}^{2}$, the LO bottom-quark-induced contributions read

$$
\begin{aligned}
\sigma_{b \bar{b} \rightarrow \tilde{b}_{1} \overline{\tilde{b}}_{1}}^{(0)}=\frac{\alpha_{\mathrm{s}}^{2} \pi C_{F}}{N_{c} s}[ & \left(\frac{m_{\tilde{g}}^{2} s\left[1-\cos \left(4 \theta_{\tilde{b}}\right)\right]}{8\left(m_{\tilde{g}}^{2} s+m_{-}^{4}\right)}-\frac{s+2 m_{\tilde{b}_{1}}^{2}}{3 s}-\frac{1+\cos \left(4 \theta_{\tilde{b}}\right)}{4}+\frac{s+2 m_{-}^{2}}{2 N_{c} s}\right) \beta \\
& \left.+\left(\frac{m_{-}^{4}+s m_{\tilde{g}}^{2}}{N_{c} s^{2}}-\frac{\left(s+2 m_{-}^{2}\right)\left[3+\cos \left(4 \theta_{\tilde{b}}\right)\right]}{8 s}\right) \log \left(\frac{1-\beta+2 m_{-}^{2} / s}{1+\beta+2 m_{-}^{2} / s}\right)\right] .
\end{aligned}
$$

As these contributions depend on the gluino mass, we give results for $m_{\tilde{g}}=200,500 \mathrm{GeV}$ and $1 \mathrm{TeV}$. From the numbers presented in table 7 it is clear that the $b \bar{b} \rightarrow \tilde{b}_{1} \overline{\tilde{b}}_{1}$ channel is always strongly suppressed, with cross sections well below $1 \%$ of the stop-like contributions.

Bottom-quark-induced $t$-channel gluino exchange also leads to $\tilde{b}_{1} \tilde{b}_{1}$ and $\overline{\tilde{b}}_{1} \overline{\tilde{b}}_{1}$ final states. The LO cross section for these processes is given by

$$
\begin{aligned}
\sigma_{b b \rightarrow \tilde{b}_{1} \tilde{b}_{1}}^{(0)}= & \frac{\alpha_{\mathrm{s}}^{2} \pi C_{F}}{N_{c} s}\left[\left(\frac{1-N_{c}}{8 N_{c}}\left[1-\cos \left(4 \theta_{\tilde{b}}\right)\right]+\frac{2 m_{\tilde{g}}^{2} s-m_{-}^{4}+\left(2 m_{\tilde{g}}^{2} s+m_{-}^{4}\right) \cos \left(4 \theta_{\tilde{b}}\right)}{8\left(m_{-}^{4}+m_{\tilde{g}}^{2} s\right)}\right) \beta\right. \\
& \left.+\left(\frac{m_{-}^{4}\left(1-\cos \left(4 \theta_{\tilde{b}}\right)\right)+4 s m_{\tilde{g}}^{2}}{4 N_{c} s\left(s+2 m_{-}^{2}\right)}-\frac{\left(s+2 m_{-}^{2}\right)\left(1-\cos \left(4 \theta_{\tilde{b}}\right)\right)}{8 s}\right) \log \left(\frac{1-\beta+2 m_{-}^{2} / s}{1+\beta+2 m_{-}^{2} / s}\right)\right],
\end{aligned}
$$

with the identical expression for the charge conjugate process $\bar{b} \bar{b} \rightarrow \overline{\tilde{b}}_{1} \overline{\tilde{b}}_{1}$. The corresponding numerical results for $\tilde{b}_{1} \tilde{b}_{1}$ production are listed in table 8 . Also the $\tilde{b}_{k} \tilde{b}_{k}$ and $\overline{\tilde{b}}_{k} \overline{\tilde{b}}_{k}$ processes are suppressed by the small bottom-quark pdfs and never exceed the per-mille level with respect to $\tilde{b}_{k} \overline{\tilde{b}}_{k}$ production.

Open Access. This article is distributed under the terms of the Creative Commons Attribution Noncommercial License which permits any noncommercial use, distribution, and reproduction in any medium, provided the original author(s) and source are credited.

\section{References}

[1] Y.A. Golfand and E.P. Likhtman, Extension of the algebra of Poincaré group generators and violation of $p$ invariance, JETP Lett. 13 (1971) 323 [Pisma Zh. Eksp. Teor. Fiz. 13 (1971) 452] [SPIRES]. 
[2] J. Wess and B. Zumino, Supergauge transformations in four-dimensions, Nucl. Phys. B 70 (1974) 39 [SPIRES].

[3] W. Beenakker, M. Krämer, T. Plehn, M. Spira and P.M. Zerwas, Stop production at hadron colliders, Nucl. Phys. B 515 (1998) 3 [hep-ph/9710451] [SPIRES].

[4] J.R. Ellis and S. Rudaz, Search for supersymmetry in toponium decays, Phys. Lett. B 128 (1983) 248 [SPIRES].

[5] LEP2 SUSY Working Group, J. Abdallah et al., Combined LEP stop and sbottom results 183-208 GeV - ALEPH, DELPHI, L3, and OPAL experiments, note LEPSUSYWG/04-02.1 at http://lepsusy.web.cern.ch/lepsusy/Welcome.html.

[6] ALEPH collaboration, A. Heister et al., Search for scalar quarks in $e^{+} e^{-}$collisions at $\sqrt{s}$ up to $209 \mathrm{GeV}$, Phys. Lett. B 537 (2002) 5 [hep-ex/0204036] [SPIRES].

[7] D0 collaboration, V.M. Abazov et al., Search for pair production of scalar bottom quarks in $p \bar{p}$ collisions at $\sqrt{s}=1.96 \mathrm{TeV}$, Phys. Rev. Lett. 97 (2006) 171806 [hep-ex/0608013] [SPIRES].

[8] CDF collaboration, T. Aaltonen et al., Search for direct pair production of supersymmetric top and supersymmetric bottom quarks in pp collisions at $\sqrt{s}=1.96 \mathrm{TeV}$, Phys. Rev. D 76 (2007) 072010 [arXiv:0707.2567] [SPIRES].

[9] D0 collaboration, V.M. Abazov et al., Search for scalar top quarks in the acoplanar charm jets and missing transverse energy final state in py collisions at $\sqrt{s}=1.96 \mathrm{TeV}$, Phys. Lett. B 665 (2008) 1 [arXiv:0803.2263] [SPIRES].

[10] D0 collaboration, V.M. Abazov et al., Search for the lightest scalar top quark in events with

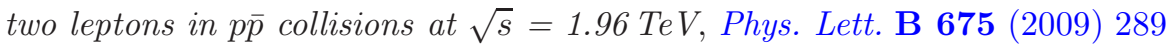
[arXiv:0811.0459] [SPIRES].

[11] CDF collaboration, T. Aaltonen et al., Search for the Production of Scalar Bottom Quarks in ppbar collisions at $\sqrt{s}=1.96 \mathrm{TeV}$, arXiv:1005.3600 [SPIRES].

[12] The ATLAS collaboration, G. Aad et al., Expected performance of the ATLAS experiment detector, trigger and physics, arXiv:0901.0512 [SPIRES].

[13] CMS collaboration, G.L. Bayatian et al., CMS technical design report, volume II: physics performance, J. Phys. G 34 (2007) 995 [SPIRES].

[14] H.P. Nilles, Supersymmetry, supergravity and particle physics, Phys. Rept. 110 (1984) 1 [SPIRES].

[15] H.E. Haber and G.L. Kane, The search for supersymmetry: probing physics beyond the standard model, Phys. Rept. 117 (1985) 75 [SPIRES].

[16] D. Berdine and D. Rainwater, Mixed top - bottom squark production at the LHC, Phys. Rev. D 72 (2005) 075003 [hep-ph/0506261] [SPIRES].

[17] G. Bozzi, B. Fuks and M. Klasen, Non-diagonal and mixed squark production at hadron colliders, Phys. Rev. D 72 (2005) 035016 [hep-ph/0507073] [SPIRES].

[18] G.L. Kane, A.A. Petrov, J. Shao and L.-T. Wang, Initial determination of the spins of the gluino and squarks at LHC, J. Phys. G 37 (2010) 045004 [arXiv:0805.1397] [SPIRES].

[19] J. Hubisz, J. Lykken, M. Pierini and M. Spiropulu, Missing energy look-alikes with $100 \mathrm{pb}^{-1}$ at the LHC, Phys. Rev. D 78 (2008) 075008 [arXiv:0805.2398] [SPIRES]. 
[20] H.K. Dreiner, M. Krämer, J.M. Lindert and B. OLeary, SUSY parameter determination at the LHC using cross sections and kinematic edges, JHEP 04 (2010) 109 [arXiv:1003.2648] [SPIRES].

[21] W. Hollik, M. Kollar and M.K. Trenkel, Hadronic production of top-squark pairs with electroweak NLO contributions, JHEP 02 (2008) 018 [arXiv:0712.0287] [SPIRES].

[22] M. Beccaria, G. Macorini, L. Panizzi, F.M. Renard and C. Verzegnassi, Stop-antistop and sbottom-antisbottom production at LHC: a one-loop search for model parameters dependence, Int. J. Mod. Phys. A 23 (2008) 4779 [arXiv:0804.1252] [SPIRES].

[23] http://www.thphys.uni-heidelberg.de/ plehn/prospino.

[24] http://people.web.psi.ch/spira/prospino/.

[25] W. Beenakker, R. Hopker, M. Spira and P.M. Zerwas, Squark and gluino production at hadron colliders, Nucl. Phys. B 492 (1997) 51 [hep-ph/9610490] [SPIRES].

[26] A. Kulesza and L. Motyka, Threshold resummation for squark-antisquark and gluino- pair production at the LHC, Phys. Rev. Lett. 102 (2009) 111802 [arXiv:0807.2405] [SPIRES].

[27] U. Langenfeld and S.-O. Moch, Higher-order soft corrections to squark hadro- production, Phys. Lett. B 675 (2009) 210 [arXiv:0901.0802] [SPIRES].

[28] A. Kulesza and L. Motyka, Soft gluon resummation for the production of gluino-gluino and squark-antisquark pairs at the LHC, Phys. Rev. D 80 (2009) 095004 [arXiv:0905.4749] [SPIRES].

[29] M. Beneke, P. Falgari and C. Schwinn, Soft radiation in heavy-particle pair production: all-order colour structure and two-loop anomalous dimension, Nucl. Phys. B 828 (2010) 69 [arXiv:0907.1443] [SPIRES].

[30] M. Beneke, P. Falgari and C. Schwinn, Soft and Coulomb gluon resummation in squark-antisquark production at the LHC, PoS (RADCOR2009) 012 [arXiv: 1001.4627] [SPIRES].

[31] W. Beenakker et al., Soft-gluon resummation for squark and gluino hadroproduction, JHEP 12 (2009) 041 [arXiv:0909.4418] [SPIRES].

[32] S. Catani, M.L. Mangano and P. Nason, Sudakov resummation for prompt photon production in hadron collisions, JHEP 07 (1998) 024 [hep-ph/9806484] [SPIRES].

[33] S. Catani, M.L. Mangano, P. Nason, C. Oleari and W. Vogelsang, Sudakov resummation effects in prompt photon hadroproduction, JHEP 03 (1999) 025 [hep-ph/9903436] [SPIRES].

[34] D. de Florian, A. Kulesza and W. Vogelsang, Threshold resummation for high-transverse-momentum Higgs production at the LHC, JHEP 02 (2006) 047 [hep-ph/0511205] [SPIRES].

[35] D. de Florian and W. Vogelsang, Threshold resummation for the inclusive hadron cross-section in p p collisions, Phys. Rev. D 71 (2005) 114004 [hep-ph/0501258] [SPIRES].

[36] D. de Florian and W. Vogelsang, Resummed cross-section for jet production at hadron colliders, Phys. Rev. D 76 (2007) 074031 [arXiv:0704.1677] [SPIRES].

[37] N. Kidonakis and R. Vogt, Next-to-next-to-leading order soft gluon corrections in top quark hadroproduction, Phys. Rev. D 68 (2003) 114014 [hep-ph/0308222] [SPIRES].

[38] A. Banfi and E. Laenen, Joint resummation for heavy quark production, Phys. Rev. D 71 (2005) 034003 [hep-ph/0411241] [SPIRES]. 
[39] R. Bonciani, S. Catani, M.L. Mangano and P. Nason, NLL resummation of the heavy-quark hadroproduction cross- section, Nucl. Phys. B 529 (1998) 424 [hep-ph/9801375] [SPIRES].

[40] N. Kidonakis and G.F. Sterman, Resummation for QCD hard scattering, Nucl. Phys. B 505 (1997) 321 [hep-ph/9705234] [SPIRES].

[41] N. Kidonakis, E. Laenen, S. Moch and R. Vogt, Sudakov resummation and finite order expansions of heavy quark hadroproduction cross sections, Phys. Rev. D 64 (2001) 114001 [hep-ph/0105041] [SPIRES].

[42] S. Moch and A. Vogt, Threshold resummation of the structure function $F_{L}$, JHEP 04 (2009) 081 [arXiv: 0902 .2342] [SPIRES].

[43] G. Grunberg, On threshold resummation of the longitudinal structure function, arXiv:0910.3894 [SPIRES].

[44] R. Akhoury, M.G. Sotiropoulos and G.F. Sterman, An operator expansion for the elastic limit, Phys. Rev. Lett. 81 (1998) 3819 [hep-ph/9807330] [SPIRES].

[45] R. Akhoury and M.G. Sotiropoulos, The large-x factorization of the longitudinal structure function, hep-ph/0304131 [SPIRES].

[46] E. Laenen, J. Smith and W.L. van Neerven, All order resummation of soft gluon contributions to heavy quark production in hadron hadron collisions, Nucl. Phys. B 369 (1992) 543 [SPIRES].

[47] S. Catani, M.L. Mangano, P. Nason and L. Trentadue, The Resummation of Soft Gluon in Hadronic Collisions, Nucl. Phys. B 478 (1996) 273 [hep-ph/9604351] [SPIRES].

[48] A.D. Martin, W.J. Stirling, R.S. Thorne and G. Watt, private communication: Parton distribution function with improved accuracy in the evolution at large $x$, based on the MSTW parametrization at the input scale [49].

[49] A.D. Martin, W.J. Stirling, R.S. Thorne and G. Watt, Parton distributions for the LHC, Eur. Phys. J. C 63 (2009) 189 [arXiv:0901.0002] [SPIRES].

[50] A. Vogt, Efficient evolution of unpolarized and polarized parton distributions with QCD-PEGASUS, Comput. Phys. Commun. 170 (2005) 65 [hep-ph/0408244] [SPIRES].

[51] A. Kulesza, G.F. Sterman and W. Vogelsang, Joint resummation in electroweak boson production, Phys. Rev. D 66 (2002) 014011 [hep-ph/0202251] [SPIRES].

[52] J.A. Aguilar-Saavedra et al., Supersymmetry parameter analysis: SPA convention and project, Eur. Phys. J. C 46 (2006) 43 [hep-ph/0511344] [SPIRES].

[53] Particle Data Group collaboration, C. Amsler et al., Review of particle physics, Phys. Lett. B 667 (2008) 1 [SPIRES].

[54] W. Porod, SPheno, a program for calculating supersymmetric spectra, SUSY particle decays and SUSY particle production at $e^{+} e^{-}$colliders, Comput. Phys. Commun. 153 (2003) 275 [hep-ph/0301101] [SPIRES].

[55] P.M. Nadolsky et al., Implications of CTEQ global analysis for collider observables, Phys. Rev. D 78 (2008) 013004 [arXiv:0802.0007] [SPIRES].

[56] R.D. Ball et al., A first unbiased global NLO determination of parton distributions and their uncertainties, Nucl. Phys. B 838 (2010) 136 [arXiv: 1002.4407] [SPIRES].

[57] W. Beenakker, M. Krämer, T. Plehn and M. Spira, SUSY particle production at the Tevatron, hep-ph/9810290 [SPIRES]. 